\title{
A comparative note on the relaxation algorithms for the linear semi-infinite feasibility problem *
}

\author{
A. Ferrer, ${ }^{\dagger} \quad$ M.A. Goberna $a^{\ddagger} \quad$ E. González-Gutiérrez ${ }^{\S}$ \\ M.I. Todorov
}

November 25, 2015

\begin{abstract}
The problem $(L F P)$ of finding a feasible solution to a given linear semi-infinite system arises in different contexts. This paper provides an empirical comparative study of relaxation algorithms for $(L F P)$. In this study we consider, together with the classical algorithm, implemented with different values of the fixed parameter (the step size), a new relaxation algorithm with random parameter which outperforms the classical one in most test problems whatever fixed parameter is taken. This new algorithm converges geometrically to a feasible solution under mild conditions. The relaxation algorithms under comparison have been implemented using the Extended Cutting Angle Method (ECAM) for solving the global optimization subproblems.
\end{abstract}

Keywords: Linear semi-infinite systems, feasibility problem, relaxation method, cutting angle method.

*This research was partially supported by MICINN of Spain, Grant MTM2014-59179C2-1-P and Sistema Nacional de Investigadores, Mexico.

†Departament de Matemàtica Aplicada I, Universitat Politècnica de Catalunya, Spain, E-Mail: alberto.ferrer@upc.edu

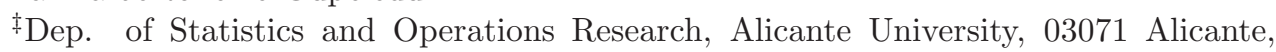
Spain. E-Mail: mgoberna@ua.es.

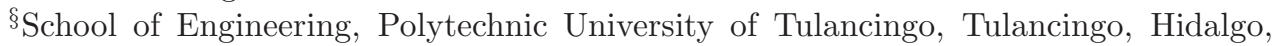
MX. E-Mail: enrique.gonzalez@upt.edu.mx

"Dep. of Physics and Mathematics, UDLAP, Puebla, MX. On leave from IMI-BAS, Sofia, BG. E-Mail: maxim.todorov@udlap.mx 


\section{Introduction}

This paper deals with linear feasibility problems of the form

$$
(L F P) \text { Find } x \in \mathbb{R}^{n} \text { such that } a(t)^{\top} x \geq b(t), \forall t \in T,
$$

where $T$ is an infinite index set, $a(t):=\left(a_{1}(t), \ldots, a_{n}(t)\right) \in \mathbb{R}^{n}$ and $b(t) \in \mathbb{R}$ for all $t \in T$. We say that $(L F P)$ is semi-infinite as the number of unknowns is finite while the number of constraints is infinite. We denote by

$$
F=\left\{x \in \mathbb{R}^{n}: a(t)^{\top} x \geq b(t), \forall t \in T\right\}
$$

the set of solutions to $(L F P)$.

Let us mention some fields where linear feasibility problems arise in a natural way. A problem like ( $L F P$ ) has to be solved to get a starting point when one applies a feasible direction method to some linear semi-infinite program (an updated list of documented applications of linear semi-infinite programming can be found in [26, Remark 1.3.3]). Some interesting applications of $(L F P)$ also include the image recovery problem [18] and the robust optimization problem [12]. In particular, the feasibility of a robust linear optimization problem can be reformulated as an example of $(L F P)$ [13]. For more recent development for robust linear multi-objective optimization problem see [23] and [24]. Observe also that any convex (possibly semi-infinite) feasibility problem

$$
\text { Find } x \in \mathbb{R}^{n} \text { such that } g_{s}(x) \leq 0, \forall s \in S,
$$

can be linearized in different ways (e.g., as in [25, (7.10)] or [19, pp. 117-118]) giving rise to a problem like $(L F P)$. Thus, numerical methods for $(L F P)$ could be used to get a starting point when solving convex programs through feasible direction methods (there exists a wide literature on the applications of convex programming). Still in the framework of convex programming, a particular instance of $(L F P)$ arises at each step of the subgradient methods (which are slower than the Newton-like methods but allow to solve nondifferentiable convex programs). Indeed, given a convex non-differentiable function $f: \mathbb{R}^{n} \rightarrow \mathbb{R}$, such methods require the computation at step $r$ of a subgradient at the current iterate $x^{r}$, i.e., they require to solve $(L F P)$ with $T$ being the domain of $f, a(t):=x^{r}-t$, and $b(t):=f\left(x^{r}\right)-f(t)$. Analogously, the computation of $\varepsilon$-subgradients and certain variational inequalities can be reformulated in terms of $(L F P)$. 
It is well-known that the linear finite feasibility problem can be solved by means of any linear programming method. Unfortunately, the same is not true when $T$ is infinite. The ellipsoid algorithm for finding a feasible point in a convex set could potentially be adapted to solve $(L F P)$, but no implementation is known up to know (even though the ingredients for the complexity analysis of such an implementation are already available [16]). The adaptation of numerical methods conceived for different problems seems also possible but not without difficulties. So, a natural way to tackle $(L F P)$ consists of reformulating it as convex finite feasibility problems by replacing the infinitely many constraints $a(t)^{\top} x \geq b(t), t \in T$, by a single convex inequality $\varphi(x) \leq 0$, where $\varphi(x):=\max _{t \in T}\left(b(t)-a(t)^{\top} x\right)$. Applying any convex programming method to minimize $\varphi$, one could either find the aimed solution of $(L F P)$ or conclude that no such solution exists. The drawback with this approach is that minimizing $\varphi$ is usually intractable as its Lipschitz constant cannot be estimated or, even worst, it is not Lipschitz continuous (unless one can replace $\mathbb{R}^{n}$ with some polytope). Another potential approach consists of extending to infinitely many sets (in this case the half-spaces $\left.\left\{x \in \mathbb{R}^{n}: a(t)^{\top} x \geq b(t)\right\}, t \in T\right)$ the Douglas-Rachford method for finite families of closed convex sets [15], but proving the convergence could be a hard task.

For all the reasons above, the unique available algorithms for solving $(L F P)$ are semi-infinite variants of the classical relaxation method introduced in 1954, independently, by Agmon and by Motzkin and Schoenberg, for the linear finite feasibility problem. It is well-known that this method either generates a finite sequence whose last element is a feasible solution or generates an infinite sequence which converges geometrically to some feasible solution. Variants of the relaxation algorithm have strongly polynomial time for special classes of the linear finite feasibility problems (see [6], [14] and references therein). The semi-infinite fixed step relaxation algorithm can be briefly described as follows: select a (relaxation) parameter $\lambda \in(0,2]$ and, if the current iterate at step $r \in \mathbb{N}$ is $x^{r} \notin F$, compute the next iterate as

$$
x^{r+1}:=x^{r}+\lambda \varepsilon_{r} \frac{a\left(t_{r}\right)}{\left\|a\left(t_{r}\right)\right\|},
$$

where $\varepsilon_{r}$ approximates the supremum $\mu_{r}$ of the distance from $x^{r}$ to the hyperplane $H_{r}=\left\{x \in \mathbb{R}^{n}: a\left(t_{r}\right)^{\top} x=b\left(t_{r}\right)\right\}$ determined by some constraint 
$a\left(t_{r}\right)^{\top} x \geq b\left(t_{r}\right), t_{r} \in T$, violated by $x^{r}: \lambda=1$ in [33] and [34], while $\lambda \in(0,2]$ in [27], [28], [29], and [30]. If $\varepsilon_{r}=\mu_{r}, x^{r+1}$ is the projection of $x^{r}$ onto $H_{r}$ when $\lambda=1$ and the symmetric of $x^{r}$ with respect to $H_{r}$ when $\lambda=2$. All the mentioned works are focused on the convergence analysis and provide few numerical examples (if any).

In this paper we propose a new relaxation algorithm where the user could select a parameter $\nu \in(0,2)$ and replace the fixed parameter $\lambda$ in (2) by some $\lambda_{r} \in[\nu, 2]$ depending on $r$. The sequence $\left\{\lambda_{r}\right\} \subset[\nu, 2]$ can be either predetermined by the user or generated at random. In all our implementations of the latter algorithm $\lambda_{r}$ is a random variable uniformly distributed on $[\nu, 2]$. This is also the first work comparing the numerical efficiency of the relaxation algorithms for $(L F P)$, with different values of the relevant parameters from the efficiency point of view: $\lambda$ in the case of relaxation with fixed step length and $\nu$ in the case of relaxation with random step length.

Section 2 contains the necessary notation, the expression of the assumptions of the convergence theorems in terms of the data. We also mention some features of the Extended Cutting Angle Method (ECAM) used to check the feasibility of the current iterate $x^{r}$ and to construct the new iterate $x^{r+1}$ (two global optimization subproblems). Section 3 shows the convergence of the new algorithm under some mild conditions while Section 4 shows its geometric convergence. Section 5 describes the numerical experiments to compare the computational efficiency of several implementations of the classical and the new relaxation algorithm, Finally, Section 6 provides the conclusions of this comparative study. For the sake of completeness we include a first appendix providing complementary information on ECAM and a second one containing a brief introduction to the performance profiles used to interpret the numerical experiments.

\section{Preliminaries}

We start this section by introducing the necessary notation. The Euclidean norm of $x \in \mathbb{R}^{n}$ is represented by $\|x\|$, the corresponding open ball centered at $x$ and radius $\varepsilon>0$ by $B_{\varepsilon}(x)$, and the zero vector by $0_{n}$. The Euclidean distance in $\mathbb{R}^{n}$ is denoted by $d$. The $L_{1}$ norm of $x \in \mathbb{R}^{n}$ is represented by $\|x\|_{1}$. Given $X \subset \mathbb{R}^{n}, \operatorname{cl} X$ and bd $X$ denote the closure and the boundary of $X$, span $X$ the linear span of $X$, aff $X$ the affine hull of $X$, conv $X$ the convex hull of $X$, and cone $X:=\mathbb{R}_{+}$conv $X$ the convex conical hull of $X \cup\left\{0_{n}\right\}$. If $X$ 
is convex, $\operatorname{dim} X$, ri $X$, and extr $X$ denote the dimension, the relative interior, and the set of extreme points of $X$, respectively. We also denote by $\mathbb{R}^{(T)}$ the space of mappings $\xi: T \rightarrow \mathbb{R}$ with finite support $\{t \in T: \xi(t) \neq 0\}$, and by $\mathbb{R}_{+}^{(T)}$ its positive cone.

The graph of a real-valued function $f$ is denoted by gph $f$ and its domain by $\operatorname{dom} f$; moreover, given $x \in \operatorname{dom} f$, the gradient and the convex subdifferential of $f$ at $x$ are denoted by $\nabla f(x)$ and $\partial f(x)$, when they exist.

We associate with $(L F P)$, corresponding to the linear system

$$
\left\{a(t)^{\top} x \geq b(t), t \in T\right\}
$$

the so-called reference cone

$$
K(a, b):=\text { cl cone }\left\{(a(t), b(t)), t \in T ;\left(0_{n},-1\right)\right\},
$$

where $a \in\left(\mathbb{R}^{n}\right)^{T}$ and $b \in \mathbb{R}^{T}$ are the functions $t \mapsto a(t)$ and $t \mapsto b(t)$, respectively. The existence theorem for linear semi-infinite systems establishes that $F \neq \emptyset$ if and only if $\left(0_{n}, 1\right) \notin K(a, b)$ while the Farkas lemma asserts that, given $F \neq \emptyset$ and $(c, d) \in \mathbb{R}^{n+1}, c^{\top} x \geq d$ holds for all $x \in F$ if and only the coefficient's vector $(c, d) \in K(a, b)$ [25, Chapter 3]. Consequently,

$$
\operatorname{aff} F=\bigcap_{(c, d) \in \mathcal{H}}\left\{x \in \mathbb{R}^{n}: c^{\top} x=d\right\}
$$

where $\mathcal{H}:=\left\{(c, d) \in \mathbb{R}^{n+1}: \operatorname{span}\{(c, d)\} \subset K(a, b)\right\}$. Then, $\operatorname{dim} F=n$ if and only if $\mathcal{H}=\left\{0_{n+1}\right\}$ if and only if $K(a, b)$ contains no line [25, Corollary 3.1.1 and Theorem 5.8]. Thus, the condition for the convergence of the relaxation algorithm with arbitrary starting point $x^{0}, \operatorname{dim} F=n$ (or the weakest one that $x^{0} \in$ aff $F$ ) can be expressed in terms of the data, but unfortunately, it can hardy be verified in practice.

We solve the global optimization subproblems in the implementations of the relaxation algorithms by means of the Extended Cutting Angle Method (ECAM in short). ECAM solves optimization problems of the form

$$
\inf \{f(x): x \in X\}
$$

where $f$ is Lipschitz continuous with known Lipschitz constant and $X \subset \mathbb{R}^{n}$ is a polytope (i.e., a bounded convex polyhedral set). We denote by $\inf _{X} f \in \mathbb{R}$ the optimal value of (3). ECAM is briefly described in Appendix 1. We shall 
use the following two lemmas to get the Lipschitz constants for the functions involved in the subproblems to be solved by the relaxation algorithms in this paper. The first lemma deals with the generation of Lipschitz continuous functions from functions of the same class while the second lemma exploits the smoothness of the functions and the convexity of their domains. The proofs can be found in the standard literature on the subject (see, e.g., [21, Chapter 12] and [32, Proposition 5.1]).

Lemma 1 Let $f_{1}, f_{2}: T \rightarrow \mathbb{R}$ be Lipschitz continuous on $T$ with constants $L_{1}, L_{2}$. Then the following statements hold:

(i) If $\sup _{T}\left|f_{i}\right| \leq M_{i}<+\infty, i=1,2$, then the product $f_{1} f_{2}$ is Lipschitz continuous on $T$ with Lipschitz modulus (the smallest Lipschitz constant) at most $M_{1} L_{2}+M_{2} L_{1}$.

(ii) If $0<m_{1} \leq \inf _{T}\left|f_{1}\right|$ and $\sup _{T}\left|f_{2}\right| \leq M_{2}<+\infty$, then, $\frac{f_{2}}{f_{1}}$ is Lipschitz continuous on $T$ with Lipschitz modulus at most $\frac{L_{2}}{m_{1}}+\frac{M_{2} L_{1}}{m_{1}^{2}}$.

With $f \in \mathcal{C}^{1}(T)$ we mean that $f$ is continuously differentiable on an open set containing $T \subset \mathbb{R}^{m}, m \in \mathbb{N}$.

Lemma 2 Let $T \subset \mathbb{R}^{m}$ be a non-singleton compact convex set and $f \in$ $\mathcal{C}^{1}(T)$. Then, $f$ is Lipschitz continuous on $T$ with Lipschitz modulus at most $\max _{T}\|\nabla f\|$.

\section{Convergence of the extended relaxation al- gorithm}

From now on we assume that $a(t) \neq 0_{n}$ for all $t \in T$, so that the function $g(\cdot, x):=a(\cdot)^{\top} x-b(\cdot)$ is well-defined for all $x \in \mathbb{R}^{n}$. Moreover, $g(\cdot, x)$ satisfies $\inf _{T} \frac{g(\cdot, x)}{\|a(\cdot)\|} \neq-\infty$ as, in the contrary, there exists a sequence $\left\{t_{k}\right\} \subset T$ such that $\frac{g\left(t_{k}, x\right)}{\left\|a\left(t_{k}\right)\right\|} \rightarrow-\infty$ as $k \rightarrow \infty$ and, taking into account that $\left\|\frac{a\left(t_{k}\right)^{\top} x}{\left\|a\left(t_{k}\right)\right\|}\right\| \leq\|x\|$, we have $\frac{b\left(t_{k}\right)}{\left\|a\left(t_{k}\right)\right\|} \rightarrow+\infty$, which in turn implies that $\frac{a\left(t_{k}\right)}{b\left(t_{k}\right)} \rightarrow 0_{n}$ as $k \rightarrow \infty$. So, $\left(0_{n}, 1\right) \in K(a, b)$ (contradiction). Consequently, the extended relaxation algorithm (ERA in short) described in Table 1, where the step length is not necessarily predetermined, is well-defined too. 
Table 1: Extended relaxation algorithm, ERA

\section{Procedure: ERA}

\section{Initialization:}

Select $M>1, \beta_{0}>0, \tau>0$ (precision), $\left.\left.\nu \in\right] 0,2\right]$;

Choose $x^{0} \in \mathbb{R}^{n}$;

$r:=0$ (set to zero initial iteration),

$\beta:=\beta_{0}$ (value for the initial $\beta$-global optimal solution),

non_stop:=true (binary variable);

\section{begin}

while (non_stop) do

Obtain (via ECAM) $\varepsilon_{r}$, a $\beta$-global optimal solution by solving the problem:

$$
\mu_{r}-\beta<\varepsilon_{r}:=\frac{b\left(t_{r}\right)-a\left(t_{r}\right)^{\top} x^{r}}{\left\|a\left(t_{r}\right)\right\|} \leq \mu_{r}:=-\inf \left\{\frac{a(t)^{\top} x^{r}-b(t)}{\|a(t)\|}: t \in T\right\}
$$

$$
\begin{aligned}
\operatorname{if}\left(\varepsilon_{r} \geq \tau\right) \text { then } & \\
& \text { if }\left(\beta<\varepsilon_{r}(M-1)\right) \text { then }
\end{aligned}
$$

Choose $\lambda_{r} \in[\nu, 2]$ (in some way);

$$
x^{r+1}:=x^{r}+\lambda_{r} \varepsilon_{r} \frac{a\left(t_{r}\right)}{\left\|a\left(t_{r}\right)\right\|}
$$

$$
r:=r+1
$$

else

$$
\begin{aligned}
& \beta:=\beta / 2 \\
& \text { endif }
\end{aligned}
$$

else

$$
\text { endif }
$$

non_stop:=false;

\section{endwhile}

return $x^{r}$, a feasible solution;

\section{end}


Before to proceed further, we shall make some comments. The parameter $\beta$ represents the accuracy level required for the subproblem (4) to be solved at step $r$, whose exact optimal value is denoted by $\mu_{r}$, while $t_{r}$ and $\varepsilon_{r}$ are, an approximation of the current optimal global solution, and a $\beta$-global approximation of the optimal value, respectively. If $\beta<\varepsilon_{r}(M-1)$ one can compute directly the new iterate; if not, $\beta$ is replaced by a smaller positive scalar of the form $\frac{\beta}{2^{k}}, k \in \mathbb{N}$, until the previous inequality holds. The necessity of enforcing $\beta<\varepsilon_{r}(M-1)$ at each step comes from the fact that this inequality guarantees that $\mu_{r} \rightarrow 0$, which is the main ingredient of the convergence proof of Theorem 6 below.

Obviously, ERA can be implemented in different ways, e.g., by taking $\lambda_{r}=\lambda$ (a fixed parameter in $[\nu, 2]$ ) for each $r=0,1,2, \ldots$ (the classical fixed step relaxation algorithm FISRA), by choosing a predetermined sequence $\left\{\lambda_{r}\right\} \subset[\nu, 2]$, or by picking up the parameter $\lambda_{r}$ at random in some subinterval of $[\nu, 2]$ (the new random step relaxation algorithm RASRA). Iteration $r$ of ERA requires a $\beta$-optimal solution of $-\inf \left\{\frac{g\left(\cdot, x^{r}\right)}{\|a(\cdot)\|}: t \in T\right\}$, where $x^{r}$ is the current iterate. This can be done via ECAM provided that these functions are Lipschitz continuous with known Lipschitz constants on a polytope $T$ contained in some Euclidean space (in most practical applications the index set $T$ is a low dimensional box, usually with $\operatorname{dim} T \in\{1,2\}$ ).

The next two results can be useful in order to apply ECAM to the subproblems of ERA. The first one involves the constants

$$
B:=\inf _{t \in T}\|a(t)\|, N:=\sup _{t \in T}\|a(t)\|, \text { and } P:=\sup _{t \in T}|b(t)| .
$$

The first two constants, $B$ and $N$, play an important role in the proof of the convergence Theorem 11, where we shall assume that $B>0$ and $N<+\infty$. Observe that $B>0$ and $N, P<+\infty$ whenever $T$ is a compact set, $a: T \rightarrow$ $\mathbb{R}^{n}$ and $b: T \rightarrow \mathbb{R}$ are continuous.

Proposition 3 Let $b, a_{1}, \ldots, a_{n}$ be Lipschitz continuous on $T \subset \mathbb{R}^{m}$, with Lipschitz constants $L_{0}, L_{1}, \ldots, L_{n}$, and assume that $B>0$ and $N, P<+\infty$. Denote $L:=\left(L_{1}, \ldots, L_{n}\right) \in \mathbb{R}^{n}$ and let $x^{r}=\left(x_{1}^{r}, \ldots, x_{n}^{r}\right) \in \mathbb{R}^{n}$. Then $\frac{g\left(\cdot, x^{r}\right)}{\|a(\cdot)\|}$ is Lipschitz continuous on $T$ with Lipschitz modulus at most

$$
\frac{1}{B}\left(L_{0}+\|L\|\left\|x^{r}\right\|\right)+\frac{N}{B^{3}}\left(P+N\left\|x^{r}\right\|\right)\|L\|_{1} .
$$


Proof. Since $g\left(\cdot, x^{r}\right)=\sum_{i=1}^{n} x_{i}^{r} a_{i}(\cdot)-b(\cdot)$ is a linear combination of $n+1$ Lipschitz continuous functions with Lipschitz constants $L_{1}, \ldots, L_{n}$ and $L_{0}$, we get that $g\left(\cdot, x^{r}\right)$ is Lipschitz continuous on $T$ with Lipschitz modulus at most $L_{0}+\|L\|\left\|x^{r}\right\|$.

By Lemma 1(i), for each $i=1, \ldots, n, a_{i}(\cdot)^{2}$ is Lipschitz continuous on $T$ with Lipschitz modulus at most $2 N L_{i}$. So, $\|a(\cdot)\|^{2}=\sum_{i=1}^{n} a_{i}(\cdot)^{2}$ is Lipschitz continuous on $T$ with Lipschitz modulus at most $2 N\|L\|_{1}$. Thus, if $t, s \in T$, we have

$$
|\|a(t)\|-\|a(s)\||=\left|\frac{\|a(t)\|^{2}-\|a(s)\|^{2}}{\|a(t)\|+\|a(s)\|}\right| \leq\left(\frac{N\|L\|_{1}}{B}\right)\|t-s\|,
$$

which shows that $\|a(\cdot)\|$ is Lipschitz continuous on $T$ with Lipschitz modulus at most $\frac{N\|L\|_{1}}{B}$.

Observe also that, given $t \in T$,

$$
\left|g\left(t, x^{r}\right)\right| \leq|b(t)|+\left\|x^{r}\right\|\|a(t)\| \leq P+N\left\|x^{r}\right\| .
$$

Now we apply Lemma $1\left(\right.$ ii) to the functions $f_{1}=\|a(\cdot)\|$ and $f_{2}=g\left(\cdot, x^{r}\right)$, with $0<B \leq \inf _{T}\left|f_{1}\right|$ and

$$
\sup _{T}\left|f_{2}\right|=\sup _{T}\left|g\left(\cdot, x^{r}\right)\right| \leq P+N\left\|x^{r}\right\|<+\infty
$$

by (7). Then we get (6).

Let us introduce two additional constants when $b, a_{1}, \ldots, a_{n} \in \mathcal{C}^{1}(T)$ and $T$ is compact:

$$
Q:=\max _{i=1, \ldots, n ; t \in T}\left\|\nabla a_{i}(t)\right\| \text { and } R:=\max _{t \in T}\|\nabla b(t)\| .
$$

Proposition 4 Let $T \subset \mathbb{R}^{m}$ be a non-singleton compact convex set, $b, a_{1}, \ldots, a_{n} \in$ $\mathcal{C}^{1}(T)$, and assume that $B>0$. Then, given $x^{r} \in \mathbb{R}^{n}, \frac{g\left(\cdot, x^{r}\right)}{\|a(\cdot)\|}$ is Lipschitz continuous on $T$ with Lipschitz modulus at most

$$
\left(\frac{N}{B^{3}}\right)\left[B\left(\left\|x^{r}\right\|_{1} Q+R\right)+\left(P+N\left\|x^{r}\right\|\right) n Q\right] .
$$

Proof. Observe that

$$
\nabla_{t} \frac{g\left(t, x^{r}\right)}{\|a(t)\|}=\frac{\|a(t)\| \nabla_{t} g\left(t, x^{r}\right)-g\left(t, x^{r}\right) \nabla(\|a(t)\|)}{\|a(t)\|^{2}} .
$$


Since $\max _{t \in T}\|a(t)\| \leq N$, then

$$
\max _{t \in T}\left\|\nabla_{t} g\left(t, x^{r}\right)\right\| \leq\left\|x^{r}\right\|_{1} Q+R
$$

and

$$
\max _{t \in T} a s\left|g\left(t, x^{r}\right)\right| \leq P+N\left\|x^{r}\right\|
$$

Since

$$
\|\nabla(\|a(t)\|)\|=\|a(t)\|^{-1}\left\|\sum_{i=1, \ldots, n} a_{i}(t) \nabla a_{i}(t)\right\| \leq n B^{-1} N Q, \forall t \in T,
$$

one has $\max _{t \in T}\|\nabla(\|a(t)\|)\| \leq n B^{-1} N Q$, which together with $\min _{t \in T}\|a(t)\|^{2} \geq$ $B^{2}$ shows that $\max _{t \in T}\left\|\nabla_{t}\left(\frac{g\left(t, x^{r}\right)}{\|a(t)\|}\right)\right\|$ is not greater than the real number in (8). Lemma 2 yields the aimed conclusion.

Example 5 In robust linear programming with uncertain constraints (see, e.g., [24, Section 3]), one assumes that the objective function $x \mapsto c^{\top} x$ is deterministic while the coefficient vectors of the $p$ given constraints take values on given (generally infinite) uncertainty sets $\mathcal{U}_{j}, j=1, \ldots, p$. The robust feasible solutions are the feasible solutions of the so-called robust counterpart problem

$$
\min \left\{c^{\top} x: a_{j}^{\top} x \geq b_{j}, \forall\left(a_{j}, b_{j}\right) \in \mathcal{U}_{j}, j=1, \ldots, p\right\} .
$$

So, computing a robust feasible solution is the linear feasibility problem

$$
(L F P) \text { Find } x \in \mathbb{R}^{n} \text { such that } a^{\top} x \geq b, \forall(a, b) \in T,
$$

where $T=\bigcup_{j=1, \ldots, p} \mathcal{U}_{j}$, which can be written as (LFP) in (1), with a:T $\rightarrow \mathbb{R}^{n}$ such that $a\left(t_{1}, \ldots, t_{n+1}\right)=\left(t_{1}, \ldots, t_{n}\right)$ and $b: T \rightarrow \mathbb{R}$ such that $b\left(t_{1}, \ldots, t_{n+1}\right)=$ $t_{n+1}$. Observe that $T$ is compact whenever $\mathcal{U}_{j}$ is compact for all $j=1, \ldots, p$.

Obviously, the projection functions $a_{i}(\cdot)$ and $b(\cdot)$ are Lipschitz continuous with Lipschitz moduli equal to 1 . Most robust decision makers choose uncertainty sets of the form

$$
\mathcal{U}_{j}:=\left(\bar{a}_{j}, \bar{b}_{j}\right)+\alpha_{j} \mathcal{U}, \quad j=1, \ldots, p,
$$

where $\left(\bar{a}_{j}, \bar{b}_{j}\right) \in \mathbb{R}^{n+1}$ are deterministic vectors and $\alpha_{j} \geq 0, j=1, \ldots, p$, while $\mathcal{U}$ denotes the closed unit ball for some norm on $\mathbb{R}^{n+1}$. For simplicity 
we consider here the affine constraint data perturbations model (9)-(10) with $\mathcal{U}=B_{1}\left(0_{n+1}\right)$. ERA is well defined provided that $a(t) \neq 0_{n}$ for all $t \in T$, i.e.,

$$
\left\|\bar{a}_{j}\right\|>\alpha_{j}, j=1, \ldots, p,
$$

or, equivalently, $B:=\inf _{j=1, \ldots, p}\left(\left\|\bar{a}_{j}\right\|-\alpha_{j}\right)>0$. If $x^{r} \in \mathbb{R}^{n}$ is the present iterate, by Proposition 3, $\frac{g\left(\cdot, x^{r}\right)}{\|a(\cdot)\|}$ is Lipschitz continuous on $T$ with Lipschitz modulus at most

$$
\frac{1}{B}\left(1+\sqrt{n}\left\|x^{r}\right\|\right)+\frac{n N}{B^{3}}\left(P+N\left\|x^{r}\right\|\right)
$$

where

$$
N=\sup _{j=1, \ldots, p}\left(\left\|\bar{a}_{j}\right\|+\alpha_{j}\right) \text { and } P=\sup _{j=1, \ldots, p} \max \left\{\left|\bar{b}_{j}-\alpha_{j}\right|,\left|\bar{b}_{j}+\alpha_{j}\right|\right\} .
$$

Other Lipschitz constants can be obtained for other norms in a similar way.

When the unit ball $\mathcal{U}$ is a polytope (e.g. for the $L_{1}$ and the $L_{\infty}$ norms), one can reformulate (LFP) in (9)-(10) as

$(L F P)$ Find $x \in \mathbb{R}^{n}$ such that $t^{\top}(x,-1) \geq 0, t \in T$,

where $T=\mathrm{conv}\left[\bigcup_{j=1, \ldots, p}\left(\left(\bar{a}_{j}, \bar{b}_{j}\right)+\alpha_{j} \operatorname{extr} \mathcal{U}\right)\right]$ is a polytope in $\mathbb{R}^{n+1}$. Observe that $Q=R=1$, but Proposition 4 does not applies as $T$ is the union of $p$ closed balls and, so, generally non-convex for $p \geq 2$.

According to [24, Theorem 4], (LFP) has solutions, i.e. $F \neq \emptyset$, whenever $\max _{j=1, \ldots, p} \alpha_{j}$ is less than the distance from the so-called hypographical set

$$
\operatorname{conv}\left\{\left(\bar{a}_{j}, \bar{b}_{j}\right), j=1, \ldots, p\right\}+\mathbb{R}_{+}\left\{\left(0_{n},-1\right)\right\}
$$

to the origin $0_{n+1}$. This distance can be computed by solving a quadratic programming problem. Unfortunately, the assumption that $\operatorname{dim} F=n$ in the convergence theorems below, which can be expressed in terms of the data as the requirement that the convex cone

$$
\operatorname{cl}\left(\sum_{i=1, . ., p} \mathbb{R}_{+} \text {cone }\left\{\left(\bar{a}_{j}, \bar{b}_{j}\right)+\alpha_{j} \mathcal{U}\right\}+\mathbb{R}_{+}\left\{\left(0_{n},-1\right)\right\}\right)
$$

contains no line, is not checkable. In other words, the user must apply ERA assuming that $\operatorname{dim} F=n$ and conclude that $\operatorname{dim} F<n$ for those feasibility problems for which the generated sequence $\left\{x^{r}\right\}$ does not converge to some feasible solution. 
Now, we shall modify the proof of the convergence of FISRA, [29, Theorem 3], in order to get the new proof of the convergence of ERA.

Theorem 6 (Convergence) Assume that $\operatorname{dim} F=n$. Let $x^{0} \in \mathbb{R}^{n}$ and $\nu \in] 0,2\left[\right.$. If for each $r=0,1,2, \ldots$ we chose an arbitrary $\lambda_{r} \in[\nu, 2], E R A$ either ends after a finite number of steps, or generates an infinite sequence $\left\{x^{r}\right\}$ converging to some element of $F$.

Proof. Observe that, if ERA generates a finite sequence, the last point is an approximate solution to $(L F P)$. So, we can assume w.l.o.g. that $\left\{x^{r}\right\}$ is an infinite sequence of infeasible points.

For each $t \in T$ we denote $H_{t}=\left\{x \in \mathbb{R}^{n}: a(t)^{\top} x=b(t)\right\}$. Given $r \in \mathbb{N}$, we have $\mu_{r}>0$, i.e., $x^{r} \notin H_{t_{r}}$ for some $t_{r} \in T$. Thus, $x^{r+1}$ belongs to the half-line emanating from $x^{r}$ in the direction of $a\left(t_{r}\right)$, with $d\left(x^{r+1}, x^{r}\right)=\lambda_{r} \varepsilon_{r}$.

By hypothesis, there exist $z \in \mathbb{R}^{n}$ and $\delta>0$ such that

$$
B_{\delta}(z) \subset F \subset\left\{x \in \mathbb{R}^{n}: a\left(t_{r}\right)^{\top} x \geq b\left(t_{r}\right)\right\}, \quad r=1,2, \ldots
$$

and $\rho_{t_{r}}:=d\left(z, H_{t_{r}}\right) \geq \delta$.

By construction, the line determined by $x^{r}$ and $x^{r+1}$, aff $\left\{x^{r}, x^{r+1}\right\}$, is orthogonal to $H_{t_{r}}$. Let $h_{r}=d\left(z\right.$, aff $\left.\left\{x^{r}, x^{r+1}\right\}\right)$. We select a coordinate system in the hyperplane aff $\left\{x^{r}, x^{r+1}, z\right\}$ such that the abscissa axis is the line aff $\left\{x^{r}, x^{r+1}\right\}$, oriented in the direction from $x^{r}$ to $x^{r+1}$, the axis of ordinates is the line orthogonal to aff $\left\{x^{r}, x^{r+1}\right\}$, oriented in such a way that $z$ belongs to the first quadrant, and the origin is located at $H_{t_{r}} \cap$ aff $\left\{x^{r}, x^{r+1}\right\}$. With this oriented system, the coordinates of the $x^{r}$ are $\left(-\varepsilon_{r}, 0\right)$, the coordinates of $x^{r+1}$ are $\left(\left(\lambda_{r}-1\right) \varepsilon_{r}, 0\right)=\left(\xi_{r} \varepsilon_{r}, 0\right)$, with $\left.\left.\lambda_{r}-1=\xi_{r} \in\right]-1,1\right]$, and the coordinates of $z$ are $\left(\rho_{t_{r}}, h_{r}\right)$, with $h_{r} \geq 0$ (the case when $\operatorname{dim} \operatorname{aff}\left\{x^{r}, x^{r+1}, z\right\}=1$ and $h_{r}=0$ is trivial). Figure 1 illustrates the notations, which are the same as in [29, Theorem 3]. 


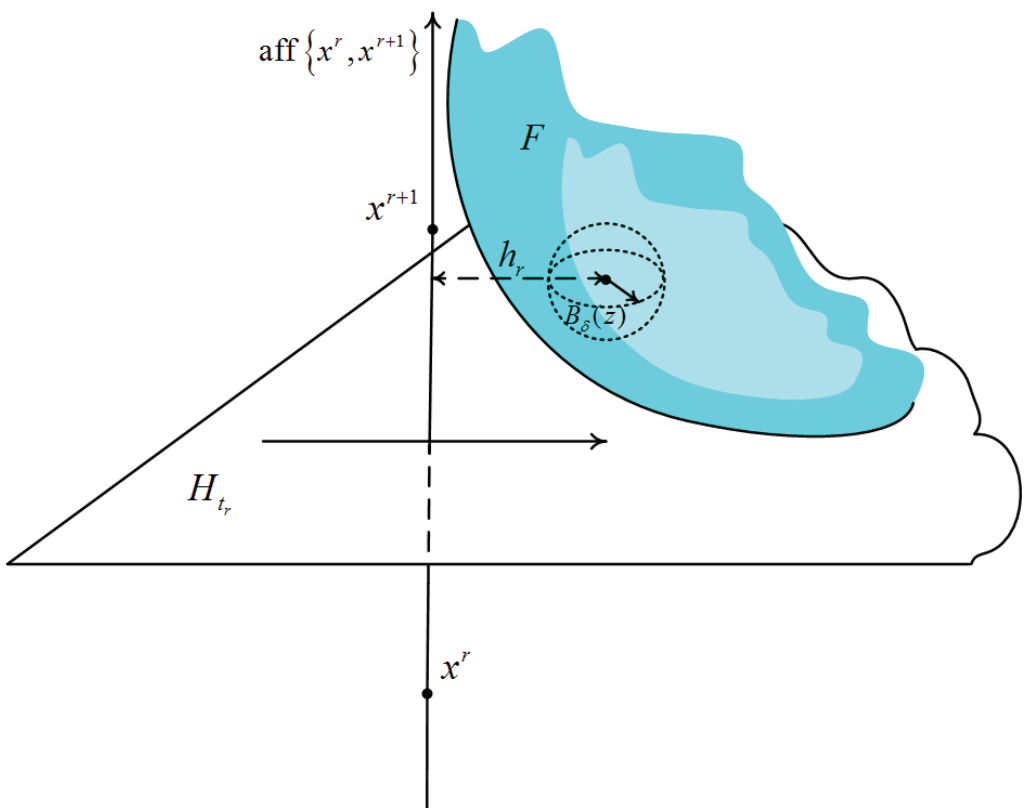

Figure 1: Coordinate system involving aff $\left\{x^{r}, x^{r+1}\right\}$ and the hyperplane $H_{t_{r}}$.

Following exactly the same steps as in the beginning of the proof of [29, Theorem 3], we obtain the following inequality

$$
\sum_{k=0}^{r-1}\left(1+\xi_{k}\right) \varepsilon_{k} \leq \frac{1}{2 \delta}\left\|x^{0}-z\right\|^{2} .
$$

Since $\xi_{k}=\lambda_{k}-1$ and $\nu \leq \lambda_{k}$ for all $k=0, \ldots, r-1$, one gets

$$
\sum_{k=0}^{r-1} \nu \varepsilon_{k} \leq \sum_{k=0}^{r-1} \lambda_{k} \varepsilon_{k} \leq \frac{1}{2 \delta}\left\|x^{0}-z\right\|^{2},
$$

which gives

$$
\sum_{k=0}^{r-1} \varepsilon_{k} \leq \frac{1}{2 \delta \nu}\left\|x^{0}-z\right\|^{2} .
$$

Defining $\eta_{r-1}:=\sum_{k=0}^{r-1} \varepsilon_{k}$, and $K:=\frac{1}{2 \nu \delta}\left\|x^{0}-z\right\|^{2}$, from (12) we get $0 \leq$ $\eta_{r-1} \leq K$ for all $r \in \mathbb{N}$. As the sequence $\left\{\eta_{r}\right\}$ is bounded and increasing, it is convergent, with $0 \leq \lim _{r} \eta_{r} \leq K$. Hence, $\sum_{r=0}^{\infty} \varepsilon_{r}$ converges as well (and $\left.\lim _{r} \varepsilon_{r}=0\right)$. 
We have that $\left|\mu_{r}-\varepsilon_{r}\right|<\beta$, for every $r=1,2, \ldots$, and require at each step that $\beta<\varepsilon_{r}(M-1)$, which is equivalent to $\beta+\varepsilon_{r}<\varepsilon_{r} M$. But, we have that $\beta+\varepsilon_{r}>\mu_{r}$, whereby

$$
\frac{\mu_{r}}{M}<\varepsilon_{r} \leq \mu_{r},
$$

i.e., $0<\mu_{r}<\varepsilon_{r} M$, so, we get $\lim _{r} \mu_{r}=0$.

From (11) we have

$$
\sum_{k=0}^{r-1} \lambda_{k} \varepsilon_{k} \leq \frac{1}{2 \delta}\left\|x^{0}-z\right\|^{2},
$$

but, from the definition of ERA, we have

$$
\lambda_{k} \varepsilon_{k}=\left\|x^{r}-x^{r+1}\right\| .
$$

So,

$$
\sum_{k=0}^{r-1}\left\|x^{r}-x^{r+1}\right\| \leq \frac{1}{2 \delta}\left\|x^{0}-z\right\|^{2}
$$

and then the series $\sum_{r=0}^{\infty}\left\|x^{r}-x^{r+1}\right\|$ converges. Therefore, $\sum_{r=0}^{\infty}\left(x^{r}-x^{r+1}\right)$ is absolutely convergent (see, e.g., [5, Theorem 26.7]), and we conclude the existence of some $\hat{x} \in \mathbb{R}^{n}$ such that $\lim _{r} x^{r}=\hat{x}$.

It remains to show that $\hat{x} \in F$. For any $t \in T$, and for all $r \in \mathbb{N}$ we have

$$
-\frac{g\left(t, x^{r}\right)}{\|a(t)\|}=\frac{b(t)-a(t)^{\top} x^{r}}{\|a(t)\|} \leq \begin{cases}\mu_{r}, & \text { if } g\left(t, x^{r}\right)<0 \\ 0, & \text { otherwise }\end{cases}
$$

Passing to the limit in (13) as $r \rightarrow \infty$ we get $\frac{b(t)-a(t)^{\top} \hat{x}}{\|a(t)\|} \leq 0$, for all $t \in T$, and this proves that $\hat{x} \in F$.

Observe that when $\operatorname{dim} F=n$ and ERA generates an infinite sequence $\left\{x^{r}\right\}$, its limit $\hat{x} \in \operatorname{bd} F$ as $x^{r} \in \mathbb{R}^{n} \backslash F$ for all $r \in \mathbb{N}$.

The next example shows that the non-degeneracy assumption that $\operatorname{dim} F=$ $n$ in Theorem 6 is not superfluous. Even more, the computational experience in Section 4 shows that the convergence is quite slow whenever the condition number of $F$ (assumed to be bounded), say cond $(F)$, defined as the quotient of the smallest width of $F$ by the greatest one, is small. Obviously, for a compact convex set set $F, \operatorname{dim} F<n$ if and only if cond $(F)=0$. 
Example 7 The simple feasibility problem

$$
(L F P) \text { Find } x \in \mathbb{R}^{2} \text { s.t. }-d(\cos t) x_{1}-c(\sin t) x_{2} \geq-c d, \forall t \in[0,2 \pi] \text {, }
$$

where $c$ and $d$ are two given positive numbers, illustrates the difficulties encountered by ERA when solving feasibility problems when $\operatorname{dim} F=n$ but cond $(F)$ is very small. It is easy to see that $F=\left\{x \in \mathbb{R}^{2}: \frac{x_{1}^{2}}{c^{2}}+\frac{x_{2}^{2}}{d^{2}} \leq 1\right\}$, with cond $(F)=\min \left\{\frac{c}{d}, \frac{d}{c}\right\}$. Assuming that ERA generates an infinite sequence $\left\{x^{r}\right\}$ whose limit $\hat{x} \neq( \pm c, 0)$, and that $0<d<c, x_{2}^{r} \neq 0$ for sufficiently large $r$ because $x_{2}^{r} \rightarrow \hat{x}_{2} \neq 0$ (as the unique points $x \in \mathrm{bd} F$ such that $x_{2}=0$ are $\left.( \pm c, 0)\right), \frac{d}{d t}\left(\frac{g\left(x^{r}, t\right)}{\|a(t)\|}\right)_{t=0}=-\frac{c}{d} x_{2}^{r}$, and

$$
\left|\frac{d}{d t}\left(\frac{g\left(x^{r}, t\right)}{\|a(t)\|}\right)_{t=0}\right|=\frac{\left|x_{2}^{r}\right|}{\operatorname{cond}(F)} \rightarrow+\infty \text { as cond }(F) \rightarrow 0 \text {. }
$$

Hence the Lipschitz modulus of $\frac{g\left(x^{r}, t\right)}{\|a(t)\|}$ tends to $+\infty$ too as cond $(F)$ tends to zero, making ECAM to become inefficient to solve the global optimization subproblems. This theoretical observation is coherent with the empirical results shown in Table 2 (see Subsection 5.2).

Consider now the limit case that $d=0$ while $c>0$. Obviously, $F=$ $\mathbb{R} \times\{0\}$ with $\operatorname{dim} F<n=2$. Recall that ERA selects at step $r$ a parameter $\lambda_{r} \in(0,2]$ and, if the current iterate is $x^{r} \notin F$, computes the next iterate by (2), with $\varepsilon_{r}$ approximating the supremum $\mu_{r}=d\left(x^{r}, H_{r}\right)$, where $H_{r}=$ $\left\{x \in \mathbb{R}^{n}: a\left(t_{r}\right)^{\top} x=b\left(t_{r}\right)\right\}$ is the hyperplane determined by some constraint violated by $x^{r}$. Consider ( LFP) with $d=0$ and take $\varepsilon_{r}=\mu_{r}$ for all $r$. Given $x^{r} \notin F$ (i.e., $x_{2}^{r} \neq 0$ ), $H_{r}=F$ (the $x$ axis), and $\operatorname{gph} g\left(\cdot, x^{r}\right)$ is the curve in red (in blue) in Figure 2 whenever $x_{2}^{r}>0\left(x_{2}^{r}<0\right.$, respectively), so that

$$
\operatorname{argmin} g\left(t, x^{r}\right)= \begin{cases}\left\{\frac{3 \pi}{2}\right\}, & \text { if } x_{2}^{r}>0, \\ \left\{\frac{\pi}{2}\right\}, & \text { if } x_{2}^{r}<0 .\end{cases}
$$




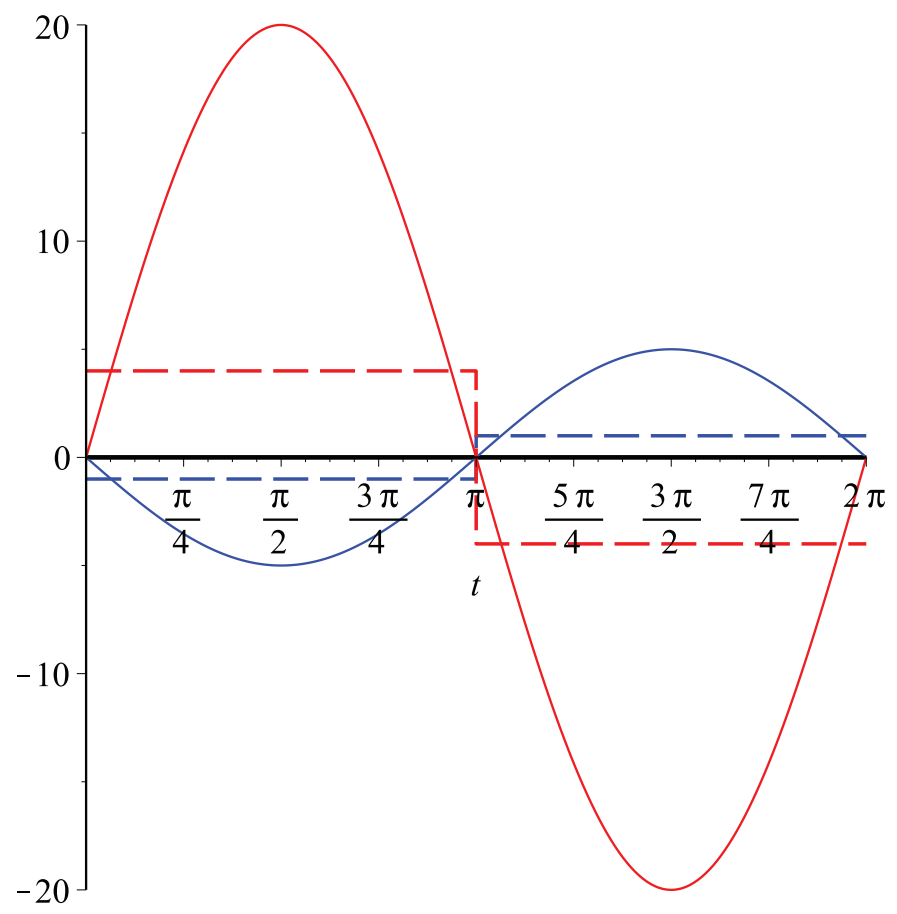

Figure 2: Graphs of the functions $g\left(\cdot, x^{r}\right)$ and $\frac{g\left(\cdot, x^{r}\right)}{\|a(\cdot)\|}$. 
Figure 2 shows the graph of the piecewise linear function $g\left(\cdot, x^{r}\right)$ (represented with dashed points) and the graph of the smooth function to be minimized at step $r, \frac{g\left(\cdot, x^{r}\right)}{\|a(\cdot)\|}$, in both cases in red (blue) whenever $x_{2}^{r}>0\left(x_{2}^{r}<0\right.$, respectively). We now apply FISRA with different choices of the step size $\lambda$ and the initial point $x^{0}$. As Figure 3 shows, the results are as follows:

i) If $\lambda=0.5$ and $x^{0}=(-7,4)$, then FISRA generates an infinite sequence $\left\{x^{r}\right\} \rightarrow x^{*} \in F$ contained in the open half-plane $x_{2}>0$.

ii) If $\lambda=1.0$ and $x^{0}=(-3,4)$, then FISRA provides a point of $x^{*} \in F$ in just one iteration.

iii) If $\lambda=1.5$ and $x^{0}=(3,4)$, then FISRA generates again an infinite sequence $\left\{x^{r}\right\} \rightarrow x^{*} \in F$, whose even (odd) terms are contained in the open half-plane $x_{2}>0\left(x_{2}<0\right.$, respectively).

iv) If $\lambda=2.0$ and $x^{0}=(7,4)$, then FISRA fails (the oscillating sequence $x^{r}=\left(7,(-1)^{r+1} 4\right)$ does not converge $)$. 


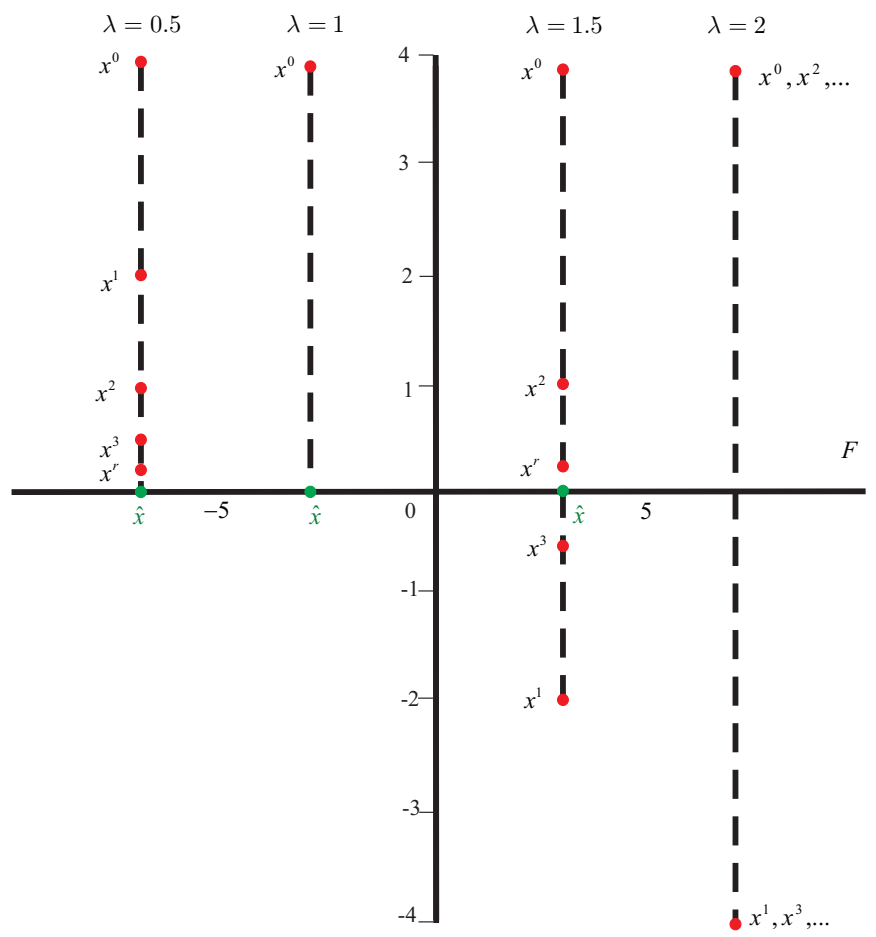

Figure 3: Different choices of the step size $\lambda$ and the initial point $x^{0}$ 
Remark 8 ERA can be conceptually adapted to the unrealistic situation in which $\operatorname{dim} F=m<n$ and the affine hull of $F$ is known, i.e., aff $F=p+V$, for a given $p \in \mathbb{R}^{n}$ and a given linear subspace $V$ of dimension $m$. We thus have:

- The translation $x=z+p$ allows us to replace $F$ with a closed convex set

$$
\bar{F}:=\left\{z \in \mathbb{R}^{n}: a(t)^{\top} z-\bar{b}(t) \geq 0, \forall t \in T\right\},
$$

with $\bar{b}(\cdot)=b(\cdot)-a(\cdot)^{\top} p$, so that $F=\bar{F}+p$ and aff $\bar{F}=V$.

- We can complete an arbitrary basis $\left\{v^{1}, \ldots, v^{m}\right\}$ of $V$ with $n-m$ linearly independent vectors $\left\{w^{m+1}, \ldots, w^{n}\right\}$ to get a basis of $\mathbb{R}^{n}$. Thus, $\mathbb{R}^{n}=V \oplus W$.

- We can find a $n \times n$ non-singular matrix $B=\left[B_{1} \mid B_{2}\right]$, with $B_{1}(n \times m)$ and $B_{2}(n \times(n-m))$, such that $z=B\left(\begin{array}{l}y^{1} \\ y^{2}\end{array}\right)$, where $y=\left(y^{1}, y^{2}\right) \in$ $\mathbb{R}^{m} \times \mathbb{R}^{n-m}$ is the vector formed by the coordinates of $z \in \mathbb{R}^{n}$ in the basis $\left\{v^{1}, \ldots, v^{m} ; w^{m+1}, \ldots, w^{n}\right\}$. Observe that $y^{2}=0$ for all $y \in V=\operatorname{aff} \bar{F}$.

- The result of replacing $z=B\left(\begin{array}{c}y^{1} \\ 0_{n-m}\end{array}\right)$ in the linear system

$$
\left\{a(t)^{\top} z-\bar{b}(t) \geq 0, \forall t \in T\right\}
$$

is the system $\left\{\widetilde{a}(t)^{\top} y_{1}-\bar{b}(t) \geq 0, \forall t \in T\right\}$, with $\widetilde{a}(\cdot)=B_{1}^{\top} a(\cdot)$.

- Then ERA allows to compute an element $\widehat{y}^{1}$ of

$$
\widetilde{F}:=\left\{y^{1} \in \mathbb{R}^{m}: \widetilde{a}(t)^{\top} z-\bar{b}(t) \geq 0, \forall t \in T\right\}
$$

as $\operatorname{dim} \widetilde{F}=\operatorname{dim} \bar{F}=m$ (i.e. $\widetilde{F}$ has full dimension in $\mathbb{R}^{m}$ ). So, $\widehat{x}:=$ $p+B\left(\begin{array}{c}\widehat{y}^{1} \\ 0_{n-m}\end{array}\right) \in F$. 


\section{Rate of convergence of ERA}

The objective of this section is to show that, taking $\lambda_{r} \in[\nu, \mu] \subset(0,2)$ for all $r \in \mathbb{N}$, the rate of convergence of ERA is geometric. To prove it we need two lemmas.

Lemma 9 [1, Lemma 2.1]Let $\lambda \in[0,2]$ and $x, y \in \mathbb{R}^{n}$ be separated by the hyperplane $H=\left\{x \in \mathbb{R}^{n}: a^{\top} x=b\right\}$, that is $a^{\top} x<b$ and $a^{\top} y \geq b$. Then

$$
\left\|x+\lambda\left(x_{H}-x\right)-y\right\|^{2} \leq\|x-y\|^{2}-\lambda(2-\lambda)\left\|x_{H}-x\right\|^{2},
$$

where $x_{H}$ is the orthogonal projection of $x$ on $H$. The equality holds if $\lambda=0$, or $\lambda=2$ and $y \in H$.

We also need the following extension of [33, Lemma 1], whose assumptions involve the smallest and greatest distances from $0_{n}$ to the set $\{a(t): t \in T\}$ introduced in (5): $B:=\inf _{t \in T}\|a(t)\| \in \mathbb{R}_{+}$and $N:=\sup _{t \in T}\|a(t)\| \in$ $\mathbb{R}_{+} \cup\{+\infty\}$, respectively.

Lemma 10 [27, Lemma 5] Assume that ERA generates an infinite sequence $\left\{x^{r}\right\}$. If $\operatorname{dim} F=n, B>0$ and $N<+\infty$, then there exists a constant $0<\gamma<1$ such that $\mu_{r} \geq \gamma d\left(x^{r}, F\right)$ for all $r=0,1,2, \ldots$.

Now, we are ready to prove the following theorem on the rate of convergence of ERA.

Theorem 11 (Geometric convergence) Let $\lambda_{r} \in[\nu, \mu] \subset(0,2)$ for all $r=0,1,2, \ldots$, with $\nu<\mu$, and assume that ERA generates an infinite sequence $\left\{x^{r}\right\}$. If $\operatorname{dim} F=n, B>0$ and $N<+\infty$, then there exist $M>1$, $0<\theta<1$, and $\bar{x} \in F$ such that $\bar{x}=\lim _{r} x^{r}$ and

$$
\left\|x^{r}-\bar{x}\right\| \leq \theta^{r}\left\|x^{0}-\bar{x}\right\|
$$

for all $r$ big enough.

Proof. From the definition of $\varepsilon_{r}$, we have $\varepsilon_{r}=\left\|x^{r}-x_{H_{t_{r}}}\right\|$, where $x_{H_{t_{r}}}$ is the orthogonal projection of $x^{r}$ on the hyperplane $H_{t_{r}}$. We know that

$$
\varepsilon_{r}>\frac{\mu_{r}}{M}, r=0,1, \ldots
$$


Let us replace $x$ by $x^{r}, y$ by $y^{r}$ and $\lambda$ by $\lambda_{r}$ in the inequality (14), where $y^{r}$ be the point in $F$ such that $\left\|x^{r}-y^{r}\right\|=d\left(x^{r}, F\right)$, that is, $y^{r}$ is the projection of $x^{r}$ on $F$. From Lemma 9, Lemma 10 and the fact that $\left\|x^{r+1}-y^{r+1}\right\|^{2} \leq$ $\left\|x^{r+1}-y^{r}\right\|^{2}$, we get

$$
\begin{aligned}
\left\|x^{r+1}-y^{r+1}\right\|^{2} & \leq\left\|x^{r+1}-y^{r}\right\|^{2} \leq\left\|x^{r}-y^{r}\right\|^{2}-\lambda_{r}\left(2-\lambda_{r}\right)\left\|x^{r}-x_{H_{t_{r}}}\right\|^{2} \\
& =\left\|x^{r}-y^{r}\right\|^{2}-\lambda_{r}\left(2-\lambda_{r}\right) \varepsilon_{r}^{2} \\
& \leq\left\|x^{r}-y^{r}\right\|^{2}-\frac{\lambda_{r}\left(2-\lambda_{r}\right) \mu_{r}^{2}}{M^{2}} \\
& \leq\left\|x^{r}-y^{r}\right\|^{2}-\frac{\lambda_{r}\left(2-\lambda_{r}\right) \gamma^{2}}{M^{2}}\left\|x^{r}-y^{r}\right\|^{2} \\
& =\left\|x^{r}-y^{r}\right\|^{2}\left(1-\lambda_{r}\left(2-\lambda_{r}\right) \gamma^{2} M^{-2}\right)
\end{aligned}
$$

Let us define $\zeta:=\min [\nu(2-\nu), \mu(2-\mu)]$. Then $0<\zeta \leq \lambda_{r}\left(2-\lambda_{r}\right) \leq$ $1, r=0,1, \ldots$ Thus, for a sufficiently large $M$ we have $0<\sigma=(1-$ $\left.\zeta \gamma^{2} M^{-2}\right)^{\frac{1}{2}}<1$ and, making use of (17) repeatedly, we get

$$
\left\|x^{r+1}-y^{r+1}\right\| \leq \sigma^{r+1}\left\|x^{0}-y^{0}\right\| .
$$

Since $\bar{x}$ and $x^{r}$ are in the ball $B_{\left\|x^{r}-y^{r}\right\|}\left(y^{r}\right)$ for each $r=0,1,2, \ldots$, we finally obtain

$$
\frac{1}{2}\left\|x^{r+1}-\bar{x}\right\| \leq\left\|x^{r+1}-y^{r+1}\right\| \leq \sigma^{r+1}\left\|x^{0}-y^{0}\right\| \leq \sigma^{r+1}\left\|x^{0}-\bar{x}\right\|,
$$

which proves the theorem for any $\theta$ such that $\sigma<\theta<1$.

Remark 12 From (18), it would be convenient estimating the smallest $\sigma$ such that (15) holds for any $\theta$ such that $\sigma<\theta<1$, for sufficiently large values of $r$. Assuming $M>1$, we can chose $M>\max \left\{1, \zeta^{\frac{1}{2}} \gamma\right\}=1$, because $\zeta \in(0,1)$ and $\gamma \in(0,1)$. This means that $\left(1-\zeta \gamma^{2}\right)^{\frac{1}{2}}<\sigma<1$.

\section{$5 \quad$ Numerical results}

In this section we present the results of numerical experiments to compare different implementations of FISRA (depending on the fixed value of $\lambda \epsilon$ $(0,2])$ and RASRA (depending on the chosen distribution for $\lambda_{r}$ ). In the latter case, we have chosen uniform distributions on intervals of the form $[\nu, 2]$, with $0<\nu<2$, but other distributions on subintervals of $(0,2]$ could be used. Observe that, for the chosen distribution of $\lambda_{r}$, RASRA converges, but the convergence could be slow as we may have $\lambda_{r}=2$. 


\section{$5.1 \quad$ Test problems}

A total of 27 linear feasibility test problems have been selected satisfying the assumption guaranteeing the convergence of the relaxation algorithms $(\operatorname{dim} F=n)$ and the conditions allowing to check the feasibility of the current iterate though ECAM ( $T$ polyhedral and Lipschitzian data functions). From the test problems, and by considering several distances from the randomly generated initial point to the origin, we have obtained 41 different test instances (see Tables 2 and 3). These distances are significative in this study because they increase the computational time. Nevertheless, in practice we don't know how far the initial point is from $F$. So, we do not consider necessary to work with initial points far from the origin since this fact increases the complexity of the functions to be optimized. In our experiments we have selected distances 10, 20 and 50, just to illustrate the difficulties associated with high distances. Instances from No. 1 to No. 12 have been generated from ellipses with decreasing condition number (cond $(F)$ ), which is indicated between parenthesis. Instances from No. 13 to No. 21 come from [31, Examples 8, 9 and 10]. Finally, instances from No. 22 to No. 41 have been generated by using the procedure described in [22]. In this latter case we can generate test problems without limitations on the number, $n$, of variables and the dimension, $m:=\operatorname{dim} T$, of the index set.

\subsection{Computational results}

The numerical experiments, which are summarized in four tables, were carried out on a PC with Processor Intel(R) Core(TM) i5-4200U CPU 1.60-2.30 $\mathrm{GHz}$ and $8 \mathrm{~GB}$ of RAM (MS Windows7 enterprise). In Tables 2 and 3, Num denotes the number assigned to the instance, Name indicates the name of the instance, and Iter and Time represent the number of iterations and the CPUTime required for obtaining a feasible solution, respectively. Table 2 describes instances with $\lambda_{r}=\lambda$ for all $r \in \mathbb{N}$ (constant sequences) while Table 3 describes instances with random values of $\lambda_{r}$. The maximum number of iterations was limited to 400 for all instances. When the algorithm needs more than 400 iterations to attain a solution of $(L F P)$, then we consider that the solver has failed in solving the problem. The failure of a solver is indicated with a star $(*)$, in the column indicating the number of iterations. 
Table 2: Fixed value of $\left.\left.\lambda_{r} \in\right] 0,2\right]$

\begin{tabular}{|c|c|c|c|c|c|c|c|c|c|c|c|c|c|c|c|c|c|c|c|}
\hline \multirow[b]{2}{*}{ Num } & \multirow[b]{2}{*}{ Name $($ cond $(F))$} & \multirow[b]{2}{*}{$n$} & \multirow[b]{2}{*}{$\mathrm{m}$} & \multicolumn{2}{|c|}{$\lambda_{r}=0.1$} & \multicolumn{2}{|c|}{$\lambda_{r}=0.4$} & \multicolumn{2}{|c|}{$\lambda_{r}=0.7$} & \multicolumn{2}{|c|}{$\lambda_{r}=1.0$} & \multicolumn{2}{|c|}{$\lambda_{r}=1.2$} & \multicolumn{2}{|c|}{$\lambda_{r}=1.5$} & \multicolumn{2}{|c|}{$\lambda_{r}=1.8$} & \multicolumn{2}{|c|}{$\lambda_{r}=2.0$} \\
\hline & & & & Iter & Time & \begin{tabular}{ll|} 
Iter \\
\end{tabular} & Time & \begin{tabular}{l|l} 
Iter \\
\end{tabular} & $\begin{array}{l}\text { Time } \\
\end{array}$ & \begin{tabular}{l|l|} 
Iter \\
\end{tabular} & Time & Iter & Time & Iter & Time & \begin{tabular}{ll|} 
Iter \\
\end{tabular} & Time & Iter & $\begin{array}{l}\text { Time } \\
\end{array}$ \\
\hline 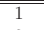 & elps.5.4.10 (0.8) & $\overline{2}$ & $\overline{11}$ & $\overline{324}$ & \begin{tabular}{|c|}
16.084 \\
\end{tabular} & 268 & 2.387 & 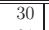 & 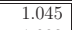 & 11 & 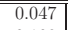 & 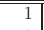 & 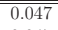 & 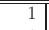 & 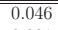 & $\bar{~} 1$ & 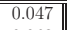 & 11 & 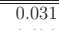 \\
\hline 2 & elps.5.4.20 & 2 & 1 & 334 & 16.240 & 70 & 2.433 & 31 & 1.092 & 3 & 0.109 & 1 & 0.047 & 1 & 0.031 & 2 & 0.063 & 2 & 0.078 \\
\hline 3 & elps.5.4.50 & 2 & 1 & 344 & 16.848 & 72 & 3.510 & 32 & 1.592 & 3 & 0.156 & 2 & 0.093 & 2 & 0.094 & 4 & 0.171 & 6 & 0.266 \\
\hline 4 & elps.5.3.10 (0.6) & 2 & 1 & 325 & 15.921 & 68 & 2.964 & 30 & 1.326 & 3 & 0.140 & 1 & 0.062 & 1 & 0.047 & 1 & 0.047 & 2 & 0.062 \\
\hline 5 & elps.5.3.20 & 2 & 1 & 332 & 15.943 & 70 & 3.105 & 31 & 1.419 & 3 & 0.156 & 1 & 0.063 & 2 & 0.078 & 2 & 0.093 & 3 & 0.125 \\
\hline 6 & elps.5.3.50 & 2 & 1 & 345 & 16.676 & 72 & 3.464 & 31 & 1.513 & 3 & 0.156 & 2 & 0.093 & 3 & 0.125 & 5 & 0.219 & 8 & 0.327 \\
\hline 7 & $\begin{array}{l}\text { elps.5.1.10 }(0.2) \\
\end{array}$ & 2 & 1 & 326 & 19.936 & 69 & 3.027 & 30 & 1.326 & 1 & 0.047 & 2 & 0.093 & 2 & 0.063 & 3 & 0.093 & 4 & 0.094 \\
\hline 8 & elps.5.1.20 & 2 & 1 & 334 & 20.546 & 70 & 4.305 & 31 & 1.903 & 2 & 0.125 & 2 & 0.109 & 3 & 0.110 & 5 & 0.187 & 9 & 0.312 \\
\hline 9 & elps.5.1.50 & 2 & 1 & 344 & 20.780 & 72 & 3.510 & 31 & 1.528 & 2 & 0.110 & 2 & 0.078 & 4 & 0.124 & 8 & 0.219 & 22 & 0.639 \\
\hline 10 & elps.5.01.10 (0.002) & 2 & 1 & 328 & 18.798 & 69 & 2.886 & 30 & 1.264 & 2 & 0.093 & 3 & 0.125 & 6 & 0.203 & 12 & 0.296 & $401^{*}$ & 72.915 \\
\hline 11 & elps.5.01.20 & 2 & 1 & 335 & 19.235 & 66 & 2.777 & 31 & 1.310 & 1 & 0.047 & 4 & 0.172 & 7 & 0.234 & 17 & 0.421 & $401^{*}$ & 74.459 \\
\hline 12 & elps.5.01.50 & 2 & 1 & 345 & 20.545 & 67 & 2.823 & 32 & 1.389 & 2 & 0.109 & 4 & 0.125 & 7 & 0.171 & 20 & 0.406 & $401^{*}$ & 68.359 \\
\hline 13 & GT14.ex8.10 & 2 & 1 & 334 & 8.666 & 70 & 1.607 & 31 & 0.702 & 1 & 0.031 & 1 & 0.031 & 2 & 0.063 & 2 & 0.047 & 3 & 0.078 \\
\hline 14 & GT14.ex8.20 & 2 & 1 & 336 & 10.327 & 70 & 1.607 & 31 & 0.733 & 6 & 0.125 & 2 & 0.047 & 2 & 0.062 & 14 & 0.109 & 4 & 0.109 \\
\hline 15 & GT14.ex8.50 & 2 & 1 & 344 & 8.830 & 72 & 1.872 & 32 & 0.795 & 3 & 0.063 & 3 & 0.078 & 3 & 0.078 & 5 & 0.109 & 8 & 0.203 \\
\hline 16 & GT14.ex9.10 & 2 & 1 & 335 & 3.994 & 70 & 0.702 & 31 & 0.296 & 1 & 0.032 & 3 & 0.062 & 7 & 0.172 & 23 & 0.343 & $401^{*}$ & 72.540 \\
\hline 17 & GT14.ex9.20 & 2 & 1 & 341 & 3.963 & 71 & 0.795 & 31 & 0.359 & 1 & 0.016 & 5 & 0.156 & 11 & 0.234 & 159 & 1.825 & $401^{*}$ & 83.132 \\
\hline 18 & GT14.ex9.50 & 2 & 1 & 346 & 4.025 & 72 & 0.671 & 31 & 0.281 & 1 & 0.015 & 3 & 0.047 & 53 & 0.437 & 25 & 0.374 & $401^{*}$ & 61.527 \\
\hline 19 & GT14.ex10.10 & 2 & 2 & 591 & 12.776 & 141 & 3.074 & 61 & 1.279 & 2 & 0.062 & 1 & 0.047 & 1 & 0.047 & 1 & 0.047 & 1 & 0.046 \\
\hline 20 & GT14.ex10.20 & 2 & 2 & 571 & 12.620 & 144 & 2.715 & 63 & 1.138 & 2 & 0.063 & & 0.031 & 1 & 0.031 & 1 & 0.031 & 1 & 0.032 \\
\hline 21 & GT14.ex10.50 & 2 & 2 & 514 & 10.218 & 149 & 2.403 & 54 & 0.904 & 3 & 0.063 & 2 & 0.031 & 2 & 0.031 & 2 & 0.031 & 2 & 0.032 \\
\hline 22 & FPftpea.20 & 3 & 1 & 335 & 19.547 & 70 & 4.337 & 31 & 1.950 & 3 & 0.218 & & 0.156 & 7 & 0.437 & 7 & 0.437 & 12 & 0.764 \\
\hline 23 & FPftpea. 20 & 5 & 1 & 336 & 20.468 & 70 & 4.290 & 31 & 1.981 & 2 & 0.171 & 2 & 0.156 & 9 & 0.593 & 7 & 0.453 & 13 & 0.826 \\
\hline 24 & FPftpea.20 & 10 & 1 & 335 & 24.149 & 70 & 4.695 & 31 & 2.278 & 2 & 0.187 & 2 & 0.156 & 13 & 0.842 & 8 & 0.531 & 12 & 0.795 \\
\hline 25 & FPftpea.20 & 15 & 1 & 335 & 26.364 & 70 & 5.102 & 31 & 2.527 & 1 & 0.125 & 2 & 0.156 & 26 & 1.731 & 9 & 0.624 & 12 & 0.827 \\
\hline 26 & FPftpea. & 25 & 1 & 335 & 27.441 & 70 & 6.770 & 31 & 2.512 & 3 & 0.374 & 1 & 0.125 & 178 & 13.541 & 12 & 0.967 & 13 & 1.061 \\
\hline 27 & FPftpea. 20 & 50 & 1 & 335 & 44.563 & 70 & 10.358 & 31 & 4.836 & 3 & 0.687 & 1 & 0.312 & 3 & 0.515 & 17 & 2.012 & 15 & 1.825 \\
\hline 28 & FPftpea. 20 & 75 & 1 & 333 & 77.113 & 71 & 14.149 & 31 & 5.632 & 2 & 0.530 & 1 & 0.312 & 3 & 0.593 & 13 & 2.137 & 14 & 2.262 \\
\hline 29 & FPftpea.20 & 100 & 1 & 336 & 74.319 & 70 & 14.118 & 31 & 6.021 & 1 & 0.281 & & 0.468 & 3 & 0.609 & 6 & 1.216 & 10 & 1.919 \\
\hline 30 & FPftpea. 20 & 500 & 1 & 335 & 350.712 & 69 & 157.716 & 29 & 27.487 & 3 & 3.214 & 2 & 2.231 & 3 & 3.198 & 5 & 5.195 & 10 & 10.155 \\
\hline 31 & FPftpea.20 & 1000 & 1 & 334 & 761.235 & 149 & 2.403 & 30 & 68.609 & 2 & 4.914 & 2 & 5.226 & 3 & 7.489 & 5 & 12.058 & 10 & 23.634 \\
\hline 32 & FPftpeaT2.20 & 3 & 2 & 297 & 39.047 & 66 & 8.128 & 34 & $\begin{array}{ll}4.118 \\
\end{array}$ & 4 & 0.577 & 2 & 0.266 & 3 & 0.374 & 5 & 0.577 & 10 & 1.092 \\
\hline 33 & FPftpeaT2.20 & 5 & 2 & 297 & 368 & 60 & 8.424 & 29 & 6.771 & 3 & 0.483 & & 0.359 & 3 & 0.718 & 5 & 0.920 & 10 & 2.059 \\
\hline 34 & FPftpeaT2.20 & 10 & 2 & 243 & 45.053 & 72 & 10.858 & 31 & 8.829 & 3 & 0.749 & & 0.577 & 3 & 0.812 & 5 & 1.482 & 10 & 2.636 \\
\hline 35 & FPftpeaT2.20 & 15 & 2 & 344 & 54.729 & 73 & 16.552 & 30 & 7.394 & 2 & 0.655 & 2 & 0.577 & 3 & 0.93 & 5 & 1.514 & 9 & 2.511 \\
\hline 36 & FPftpeaT2.20 & 25 & 2 & 291 & 73.051 & 73 & 21.856 & 26 & 6.302 & 3 & 0.920 & 2 & 0.733 & 3 & 1.061 & 5 & 1.622 & 9 & 2.964 \\
\hline 37 & FPftpeaT2.20 & 50 & 2 & 270 & 87.807 & 64 & 25.007 & 30 & 9.782 & 3 & 1.107 & 2 & 1.07 & 3 & 1.17 & 5 & 2.215 & 9 & 3.744 \\
\hline 38 & FPftpeaT2.20 & 75 & 2 & 254 & 114.344 & 61 & 28.361 & 25 & 12.277 & 2 & 1.186 & 2 & 1.092 & 3 & 1.607 & 5 & 2.589 & 9 & 4.353 \\
\hline 39 & FPftpeaT2.20 & 100 & 2 & 283 & 160.884 & 61 & 33.384 & 26 & 14.398 & 1 & 0.780 & 2 & 1.326 & 1 & 0.047 & 5 & 2.871 & 9 & 5.070 \\
\hline 40 & FPftpeaT2.20 & 500 & 2 & 262 & 641.210 & 61 & 133.295 & 26 & 55.443 & 1 & 3.198 & 2 & 5.148 & 1 & 0.031 & 5 & 11.435 & 10 & 22.199 \\
\hline 41 & FPftpeaT2.20 & 1000 & 2 & 282 & 1324.955 & 54 & 2374.227 & 27 & 125.783 & 3 & 16.723 & 2 & 11.279 & 3 & 15.584 & 5 & 25.818 & 10 & 49.437 \\
\hline
\end{tabular}


Table 3: Random values of $\lambda_{r} \in[\nu, 2]$ for fixed $\nu$

\begin{tabular}{|c|c|c|c|c|c|c|c|c|c|c|c|c|c|}
\hline \multirow{2}{*}{ Num } & \multirow[b]{2}{*}{ Name $($ cond $(F))$} & \multirow[b]{2}{*}{$n$} & \multirow[b]{2}{*}{$\mathrm{m}$} & \multicolumn{2}{|c|}{$\nu=0.01$} & \multicolumn{2}{|c|}{$\nu=0.4$} & \multicolumn{2}{|c|}{$\nu=1.0$} & \multicolumn{2}{|c|}{$\nu=1.5$} & \multicolumn{2}{|c|}{$\nu=1.9$} \\
\hline & & & & Iter & Time & Iter & Time & Iter & Time & Iter & Time & Iter & Time \\
\hline 1 & "elps.5.4.10(0.8) & 2 & 1 & 2 & 0.094 & 1 & 0.031 & 1 & $\overline{0.047}$ & 1 & 0.031 & 1 & $\overline{0.031}$ \\
\hline 2 & elps.5.4.20 & 2 & 1 & 1 & 0.078 & 2 & 0.094 & 2 & 0.078 & 2 & 0.093 & 2 & 0.094 \\
\hline 3 & elps.5.4.50 & 2 & 1 & 3 & 0.156 & 4 & 0.172 & 1 & 0.047 & 3 & 0.125 & 5 & 0.202 \\
\hline 4 & elps.5.3.10 (0.6) & 2 & 1 & 1 & 0.046 & 1 & 0.047 & 1 & 0.047 & 1 & 0.047 & 1 & 0.047 \\
\hline 5 & elps.5.3.20 & 2 & 1 & 1 & 0.062 & 3 & 0.063 & 2 & 0.078 & 3 & 0.109 & 3 & 0.094 \\
\hline 6 & elps.5.3.50 & 2 & 1 & 2 & 0.094 & 2 & 0.078 & 3 & 0.109 & 4 & 0.156 & 6 & 0.203 \\
\hline 7 & elps.5.1.10 (0.2) & 2 & 1 & 2 & 0.156 & 2 & 0.125 & 3 & 0.141 & 3 & 0.124 & 4 & 0.141 \\
\hline 8 & elps.5.1.20 & 2 & 1 & 3 & 0.125 & 2 & 0.125 & 3 & 0.125 & 5 & 0.187 & 7 & 0.265 \\
\hline 9 & elps.5.1.50 & 2 & 1 & 5 & 0.266 & 4 & 0.171 & 5 & 0.172 & 8 & 0.234 & 12 & 0.359 \\
\hline 10 & elps.5.01.10 (0.002) & 2 & 1 & 6 & 0.515 & 6 & 0.468 & 4 & 0.281 & 9 & 0.452 & 23 & 0.843 \\
\hline 11 & elps.5.01.20 & 2 & 1 & 5 & 0.373 & 7 & 0.233 & 6 & 0.233 & 13 & 0.414 & 57 & 1.282 \\
\hline 12 & elps.5.01.50 & 2 & 1 & 5 & 0.482 & 7 & 0.289 & 2 & 0.115 & 13 & 0.378 & 42 & 1.038 \\
\hline 13 & GT14.ex8.10 & 2 & 1 & 2 & 0.094 & 2 & 0.047 & 1 & 0.031 & 1 & 0.031 & 1 & 0.031 \\
\hline 14 & GT14.e & 2 & 1 & 4 & 0.171 & 2 & 0.063 & 1 & 0.031 & 2 & 0.062 & 3 & 0.094 \\
\hline 15 & GT14.ex8.50 & 2 & 1 & 5 & 0.172 & 4 & 0.109 & 1 & 0.031 & 3 & 0.078 & 6 & 0.156 \\
\hline 16 & GT14.ex9.10 & 2 & 1 & 5 & 0.219 & 5 & 0.078 & 4 & 0.109 & 12 & 0.187 & 76 & 0.936 \\
\hline 17 & GT14.ex9.20 & 2 & 1 & 6 & 0.266 & 8 & 0.187 & 10 & 0.218 & 16 & 0.281 & 68 & 1.014 \\
\hline 18 & GT14.ex9.50 & 2 & 1 & 30 & 0.390 & 4 & 0.125 & 10 & 0.265 & 14 & 0.359 & 82 & 1.435 \\
\hline 19 & GT14.ex10.10 & 2 & 2 & 1 & 0.016 & 2 & 0.047 & 2 & 0.046 & 2 & 0.063 & 2 & 0.031 \\
\hline 20 & GT14.ex10.20 & 2 & 2 & 4 & 0.140 & 3 & 0.094 & 2 & 0.046 & 2 & 0.047 & 2 & 0.063 \\
\hline 21 & GT14.ex10.50 & 2 & 2 & 32 & 0.437 & 30 & 0.390 & 4 & 0.094 & 4 & 0.078 & 10 & 0.265 \\
\hline 22 & FPftpea.20 & 3 & 1 & 6 & 0.374 & 3 & 0.156 & 5 & 0.219 & 7 & 0.296 & 9 & 0.359 \\
\hline 23 & FPftpea.20 & 5 & 1 & 9 & 0.582 & 7 & 0.390 & 5 & 0.374 & 6 & 0.328 & 10 & 0.530 \\
\hline 24 & $\mathrm{FPft}_{\mathrm{H}}$ & 10 & 1 & 4 & 0.344 & 4 & 0.249 & 5 & 0.328 & 4 & 0.312 & 8 & 0.468 \\
\hline 25 & FPftpea.20 & 15 & 1 & 4 & 0.359 & 1 & 0.093 & 3 & 0.219 & 5 & 0.327 & 8 & 0.593 \\
\hline 26 & FPftpea.20 & 25 & 1 & 6 & 0.437 & 3 & 0.265 & 3 & 0.281 & 5 & 0.421 & 8 & 0.655 \\
\hline 27 & FPftpea.20 & 50 & 1 & 7 & 0.999 & 4 & 0.515 & 1 & 0.156 & 3 & 0.358 & 8 & 0.952 \\
\hline 28 & FPftpea.20 & 75 & 1 & 3 & 0.570 & 3 & 0.581 & 3 & 0.560 & 5 & 0.893 & 8 & 1.313 \\
\hline 29 & FPft & 100 & 1 & 1 & 0.297 & 3 & 0.639 & 4 & 0.796 & 5 & 1.045 & 8 & 1.545 \\
\hline 30 & FPftpea.20 & 500 & 1 & 2 & 2.652 & 3 & 3.104 & 5 & 5.148 & 4 & 4.259 & 8 & 7.972 \\
\hline 31 & FPftpea.20 & 1000 & 1 & 1 & 3.136 & 5 & 13.088 & 2 & 5.476 & 6 & 15.693 & 8 & 20.436 \\
\hline 32 & FPftpeaT2.20 & 3 & 2 & 3 & 0.515 & 3 & 0.437 & 4 & 0.546 & 4 & 0.499 & 8 & 1.014 \\
\hline 33 & FPftpeaT2.20 & 5 & 2 & 17 & 2.949 & 15 & 2.231 & 3 & 0.452 & 5 & 0.858 & 8 & 1.544 \\
\hline 34 & FPftr & 10 & 2 & 8 & 2.144 & 2 & 0.586 & 3 & 1.023 & 5 & 1.347 & 8 & 2.266 \\
\hline 35 & FPftpeaT2.20 & 15 & 2 & 3 & 1.264 & 4 & 1.030 & 3 & 0.936 & 6 & 1.825 & 8 & 2.527 \\
\hline 36 & FPftpeaT2.20 & 25 & 2 & 3 & 1.342 & 4 & 1.435 & 1 & 0.437 & 4 & 1.357 & 7 & 3.089 \\
\hline 37 & FPftpeaT2.20 & 50 & 2 & 4 & 2.932 & 3 & 2.138 & 4 & 2.979 & 4 & 2.715 & 8 & 5.288 \\
\hline 38 & FPftpeaT2.20 & 75 & 2 & 4 & 3.636 & 1 & 1.077 & 3 & 3.011 & 4 & 3.853 & 7 & 7.191 \\
\hline 39 & FPftpeaT2.20 & 100 & 2 & 2 & 3.276 & 4 & 4.836 & 5 & 5.336 & 5 & 5.756 & 9 & 10.655 \\
\hline 40 & FPftpeaT2.20 & 500 & 2 & 2 & 10.421 & 3 & 15.943 & 23 & 15.023 & 5 & 24.819 & 8 & 46.442 \\
\hline 41 & FPftpeaT2.20 & 1000 & 2 & 3 & 29.421 & 2 & 24.570 & 2 & 34.648 & 5 & 59.717 & 8 & 66.471 \\
\hline
\end{tabular}


The results shown in Tables 2 and 3 are compared in Tables 4 and 5. The precise meaning of the entries in the latter tables, $\rho_{s}(1)$ (probability of success in solving a problem) and $\rho_{s}^{*}$ (probability of win over the rest) is explained in the Appendix. For the sake of brevity and clarity, we have just included two figures, Figures 4 and 5 (with different scales in the axis of abscissas, corresponding to the No. of iterations), which plot the performance profile of the results, for the number of iterations, for FISRA and for RASRA.

Table 4: Results for fixed value of $\lambda_{r}$

\begin{tabular}{|c|rr|rr|}
\hline \multirow{2}{*}{$\lambda_{r}$} & \multicolumn{2}{|c|}{ Time } & \multicolumn{2}{|c|}{ Iter } \\
\cline { 2 - 5 } & $\rho_{s}(1)$ & $\rho_{s}^{*}$ & $\rho_{s}(1)$ & $\rho_{s}^{*}$ \\
\hline \hline 0.1 & $0.0 \%$ & $100.0 \%$ & $0.0 \%$ & $100.0 \%$ \\
0.4 & $2.4 \%$ & $100.0 \%$ & $0.0 \%$ & $100.0 \%$ \\
0.7 & $0.0 \%$ & $100.0 \%$ & $0.0 \%$ & $100.0 \%$ \\
1.0 & $26.2 \%$ & $100.0 \%$ & $21.4 \%$ & $100.0 \%$ \\
1.2 & $57.14 \%$ & $100.0 \%$ & $71.43 \%$ & $100.0 \%$ \\
1.5 & $14.3 \%$ & $100.0 \%$ & $51.2 \%$ & $100.0 \%$ \\
1.8 & $7.1 \%$ & $100.0 \%$ & $0.0 \%$ & $100.0 \%$ \\
2.0 & $9.5 \%$ & $85.7 \%$ & $0.0 \%$ & $85.7 \%$ \\
\hline
\end{tabular}

Table 5: Results for random values of $\lambda_{r}$

\begin{tabular}{|c|rr|rr|}
\hline \multirow{2}{*}{$\nu$} & \multicolumn{2}{|c|}{ Time } & \multicolumn{2}{c|}{ Iter } \\
\cline { 2 - 5 } & $\rho_{s}(1)$ & $\rho_{s}^{*}$ & $\rho_{s}(1)$ & $\rho_{s}^{*}$ \\
\hline \hline 0.01 & $23.8 \%$ & $100.0 \%$ & $40.5 \%$ & $100.0 \%$ \\
0.4 & $40.5 \%$ & $100.0 \%$ & $40.5 \%$ & $100.0 \%$ \\
1.0 & $35.7 \%$ & $100.0 \%$ & $45.2 \%$ & $100.0 \%$ \\
1.5 & $11.9 \%$ & $100.0 \%$ & $14.3 \%$ & $100.0 \%$ \\
1.9 & $0.0 \%$ & $100.0 \%$ & $0.0 \%$ & $100.0 \%$ \\
\hline
\end{tabular}




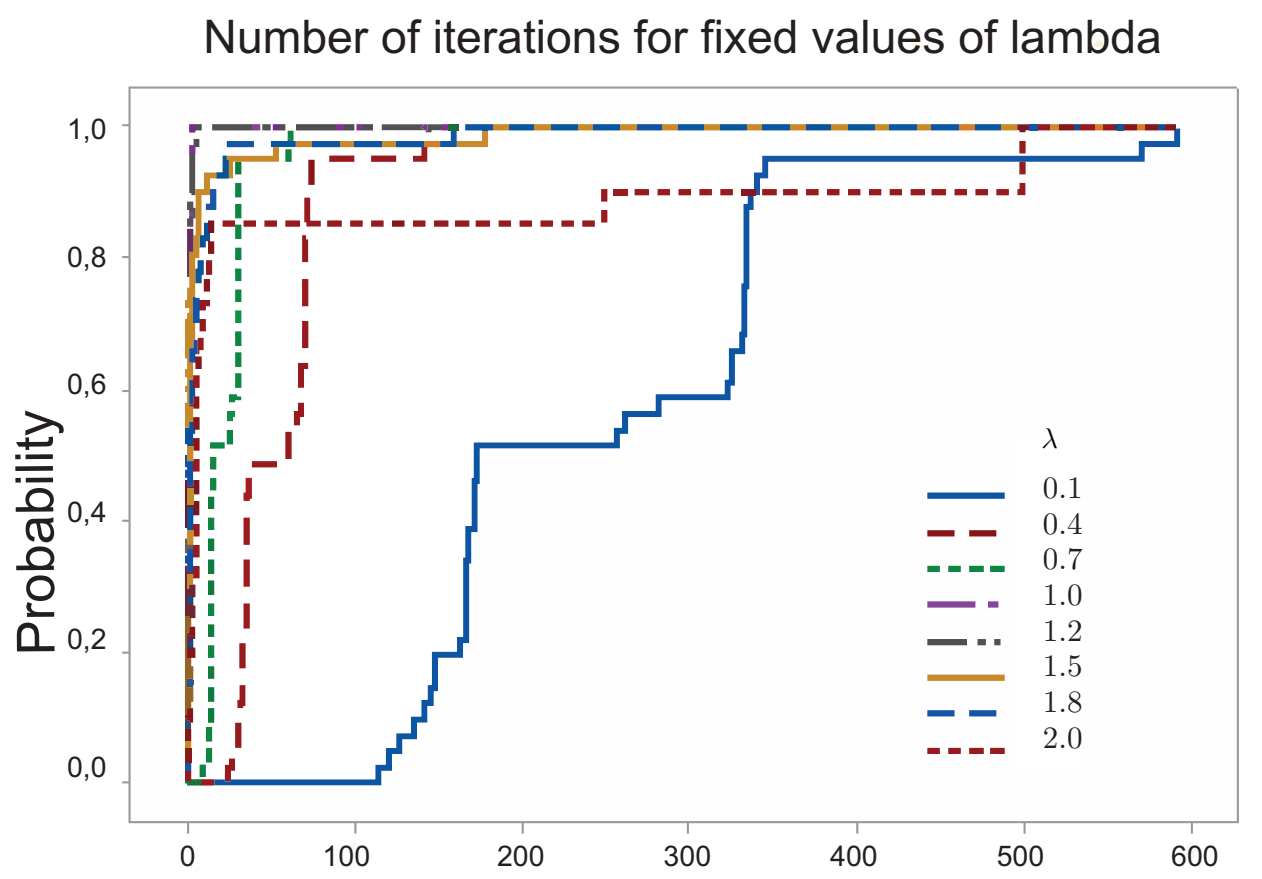

Figure 4: Iterations for fixed values of $\lambda_{r}$. 
Number of iterations for random values of lambda

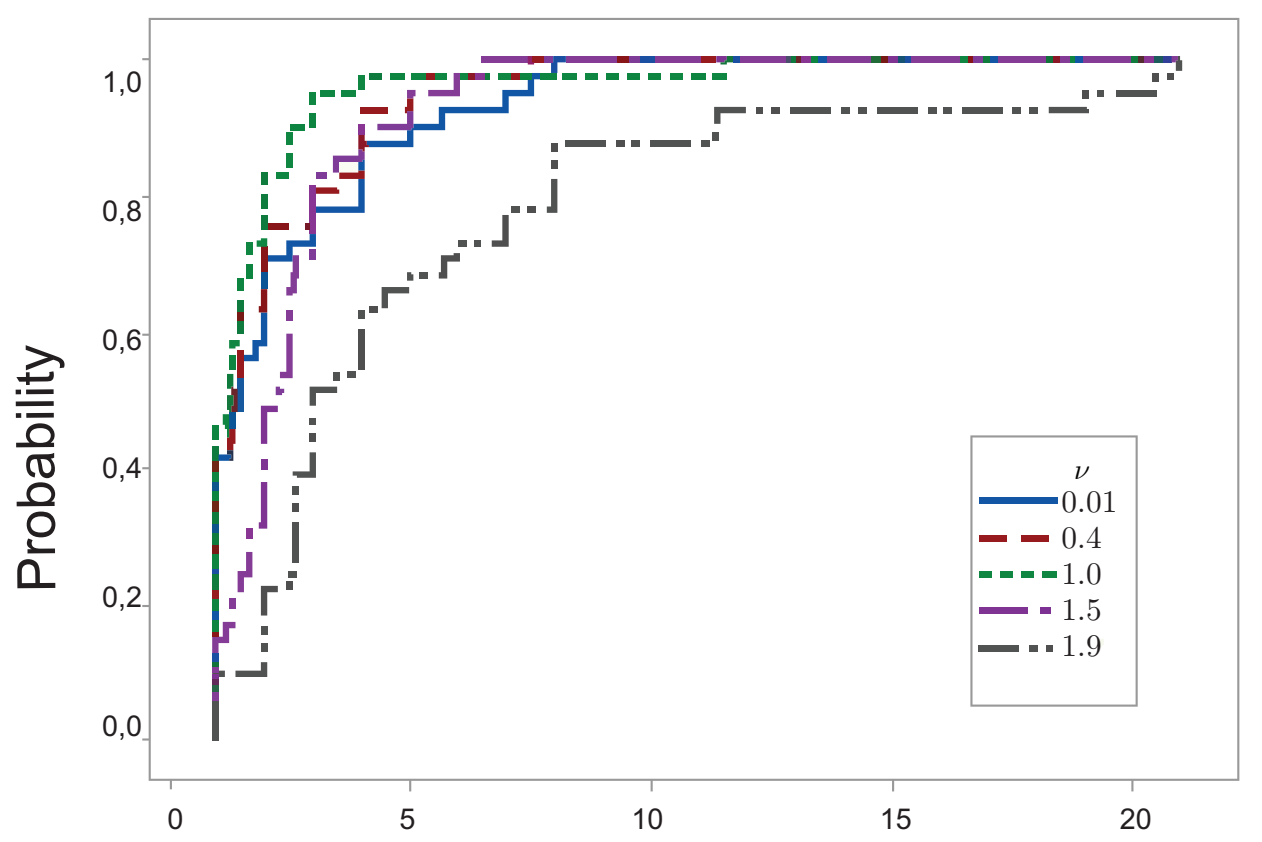

Figure 5: Iterations for random values of $\lambda_{r}$. 


\section{Conclusions}

This paper reports on the implementation of the relaxation algorithm ERA for solving $(L F P)$ which combines different step size iterations with ECAM. It is clear that the main computational difficulty to solve semi-infinite feasibility problems comes from the non-convex optimization problems that must be solved efficiently at each iteration. An innovation of this paper consists of tackling these hard global optimization subproblems with the so-called Cutting Angle Method, an efficient global optimization procedure for solving Lipschitz programming problems. Two variants of ERA with fixed and random step sizes, FISRA and RASRA, have been implemented in $\mathrm{C}++$ and run on Visual Studio 2013.

The preliminary numerical considerations are as follows. From the summary results of Tables 2 and 3, we can conclude that, in general, the number of iterations needed to attain a solution of $(L F P)$ is lower for RASRA than for FISRA. Tables 4 and 5 (and Figures 4 and 5 ) show the probability of win of each implementation over the rest and the probability of success in solving a problem. As we can see in Table 2, FISRA with constant $\lambda_{r}=2.0$ fails in solving six of the instances (i.e., it only solves the $85.7 \%$ of the instances). So, we can deduce that the random election of $\lambda_{r}$ is a more stable procedure in the sense that it solves the $100 \%$ of the instances. Nevertheless, when we consider the best case for RASRA, i.e. $\nu=0.4$, and the best case for FISRA, i.e., $\lambda_{r}=1.2$, then FISRA uses less iterations than RASRA (observe that the best fixed step size for FISRA, $\lambda_{r}=1.2$, is the middle point of the best interval $[0.4,2]$ for the random variable $\lambda_{r}$ in RASRA). Indeed, by using the corresponding performance profiles to compare the best cases, FISRA with $\lambda_{r}=1.2$ and RASRA with $\nu=0.4$ we obtain that the probability of win for fixed value of $\lambda_{r}=1.2$ is $95.1 \%$ and the probability of win for $\nu=0.4$ is $24.4 \%$.

The results obtained in the reported experiments are promising enough to suggest that suitable implementations of RASRA, which combines a relaxation method that uses random election of $\lambda_{r}$ together ECAM, could outperform FISRA for solving semi-infinite feasibility problems. In particular, the above empirical observations suggest to replace the uniform distribution of RASRA used in this paper with unimodal symmetric distributions on intervals of the form $[1.2-\varepsilon, 1.2+\varepsilon]$, for small values of $\varepsilon>0$. This could be object of further empirical studies. 


\section{Appendix: Extended Cutting Angle Method}

The Extended Cutting Angle Method (ECAM in short) due to Beliakov solves very hard optimization problems of the form

$$
\inf \{f(x): x \in X\} \text {, }
$$

where $f$ is Lipschitz continuous and $X$ is a polytope. For simplicity, we assume that $\operatorname{dim} X=n$. Since any full dimensional polytope can be expressed as the finite union of non-overlapping simplices, $X$ will be a simplex in this appendix.

In ECAM the objective function is optimized by building a sequence of piecewise linear underestimates. ECAM is inspired in the classical Cutting Plane method by Kelley [35] and Cheney and Golstein [17] to solve linearly constrained convex programs of the form (3), where $X$ is the solution set of a given linear system and $f: \mathbb{R}^{n} \rightarrow \mathbb{R}$ is convex. Since $f$ is lower semicontinuous, it is the upper envelope of the set of all its affine minorants, i.e.

$$
f=\sup \{h: h \text { affine function, } h \leq f\} .
$$

Indeed, it is enough to consider in (20) the affine functions of the form $h(x)=$ $f(z)+\langle u, x-z\rangle$, where $u \in \partial f(z)$, the graph of $h$ being a hyperplane which supports the epigraph of $f$ at $(z, f(z))$. Let $x^{1}, \ldots, x^{k} \in X$ be given and consider the affine functions $h^{j}(x)=f\left(x^{j}\right)+\left\langle u^{j}, x-x^{j}\right\rangle$, for some $u^{j} \in$ $\partial f\left(x^{j}\right), j=1, \ldots, k$. The function

$$
f_{k}:=\max _{j=1, \ldots, k} h^{j}
$$

is a convex piecewise affine underestimate of the objective function $f$, in other words, a polyhedral convex minorant of $f$. The $k$-th iteration of the Cutting Plane method consists of computing an optimal solution $x^{k+1}$ of the approximating problem inf $\left\{f_{k}(x): x \in X\right\}$ which results of replacing $f$ with $f_{k}$ in (3) or, equivalently, solving the linear programming problem in $\mathbb{R}^{n+1}$

$$
\inf \left\{x_{n+1}: x \in X, x_{n+1} \geq h^{j}(x), j=1, \ldots, k\right\},
$$

where $x=\left(x_{1}, \ldots, x_{n}\right)$. Then the next underestimate of $f$,

$$
f_{k+1}:=\max \left\{f_{k}, h^{k+1}\right\},
$$


is a more accurate approximation to $f$, and the method iterates.

The Generalized Cutting Plane method for (3), where $f: \mathbb{R}^{n} \rightarrow \mathbb{R}$ is now a non-convex function while $X=\left\{x \in \mathbb{R}_{+}^{n}: \sum_{i=1}^{n} x_{i}=1\right\}$ is the unit simplex, follows the same script, except that the underestimate $f_{k}$ is built using the so-called $H$-subgradients (see [36]) instead of ordinary subgradients, so that minimizing $f_{k}$ on $S$ is no longer a convex problem. The Cutting Angle method ([3],[4]), of which ECAM is a variant, is an efficient numerical method for minimizing the underestimates when $f$ belongs to certain class of abstract convex functions. Assume that $f$ is Lipschitz continuous with Lipschitz constant $M>0$ and take a scalar $\gamma \geq M$. Let $x^{1}, \ldots, x^{k} \in S$ be given. For $j=1, \ldots, k$, we define the support vector $l^{j} \in \mathbb{R}^{n}$ by

$$
l_{i}^{j}:=\frac{f\left(x^{j}\right)}{\gamma}-x_{i}^{j}, i=1, \ldots, n,
$$

and the support function $h^{j}$ by

$$
h^{j}(x):=\min _{i=1, \ldots, n}\left(f\left(x^{j}\right)-\gamma\left(x_{i}^{j}-x_{i}\right)\right)=\min _{i=1, \ldots, n} \gamma\left(l_{i}^{j}+x_{i}\right) .
$$

Since the functions $h^{j}$ are concave piecewise affine underestimates of $f$ (i.e. polyhedral concave minorants of $f$ ), the underestimate $f_{k}$ defined in (21) is now a saw-tooth underestimate of $f$ and its minimization becomes a hard problem as (22) is no longer a linear program. ECAM locates the set $V^{k}$ of all local minima of the function $f_{k}$ which, after sorting, yields the set of global minima of $f_{k}$ (see [9] and [10] for additional information). A global minimum $x^{k+1}$ of $f_{k}$ is aggregated to the set $\left\{x^{1}, \ldots, x^{k}\right\}$ and the method iterates with $f_{k+1}:=\max \left\{f_{k}, h^{k+1}\right\}$.

As shown in $[9,10]$, a necessary and sufficient condition for a point $x^{*} \in$ ri $X$ to be a local minimizer of $f_{k}$ given by $(25),(21)$ is that there exist an index set $J=\left\{k_{1}, k_{2}, \ldots, k_{n+1}\right\}$, such that

$$
d=f_{k}\left(x^{*}\right)=\gamma\left(l_{1}^{k_{1}}+x_{1}^{*}\right)=\gamma\left(l_{2}^{k_{2}}+x_{2}^{*}\right)=\ldots=\gamma\left(l_{n}^{k_{n+1}}+x_{n+1}^{*}\right),
$$

and $\forall i \in\{1, \ldots, n+1\}$,

$$
\left(l_{i}^{k_{i}}+x_{i}^{*}\right)<\left(l_{j}^{k_{i}}+x_{j}^{*}\right), j \neq i .
$$

Let $x^{*}$ be a local minimizer of $f_{k}$, which corresponds to some index set $J$ satisfying the above conditions. Form the ordered combination of the 
support vectors $L=\left\{l^{k_{1}}, l^{k_{2}}, \ldots, l^{k_{n+1}}\right\}$ that corresponds to $J$. It is helpful to represent this combination with a matrix $L$ whose rows are the support vectors $l^{k_{i}}$ :

$$
L:=\left(\begin{array}{cccc}
l_{1}^{k_{1}} & l_{2}^{k_{1}} & \ldots & l_{n+1}^{k_{1}} \\
l_{1}^{k_{2}} & l_{2}^{k_{2}} & \ldots & l_{n+1}^{k_{2}} \\
\vdots & \vdots & \ddots & \vdots \\
l_{1}^{k_{n+1}} & l_{2}^{k_{n+1}} & \ldots & l_{n+1}^{k_{n+1}}
\end{array}\right)
$$

so that its components are given by $L_{i j}=\frac{f\left(x^{k_{i}}\right)}{\gamma}-x_{j}^{k_{i}}$.

Let the support vectors $l^{k}, k=1, \ldots, K$ be defined as in (24). Let $x^{*}$ denote a local minimizer of $f_{k}$ and $d=f_{k}\left(x^{*}\right)$. Then the matrix (26) corresponding to $x^{*}$ enjoys the following properties (see [10]):

1) $\forall i, j \in\{1, \ldots, n+1\}, i \neq j: l_{i}^{k_{j}}>l_{i}^{k_{i}}$,

2) $\forall r \notin\left\{k_{1}, k_{2}, \ldots, k_{n+1}\right\} \exists i \in\{1, \ldots, n+1\}: L_{i i}=l_{i}^{k_{i}} \geq l_{i}^{r}$,

3) $d=\frac{\gamma}{n+1}(\operatorname{Trace}(L)+1)$, and

4) $x_{i}^{*}=\frac{d}{\gamma}-l_{i}^{k_{i}}, i=1, \ldots, n+1$.

Property 1 reads that the diagonal elements of the matrix $L$ are dominated by their respective columns, and Property 2 reads that no support vector $l^{r}$ (which is not part of $L$ ) strictly dominates the diagonal of $L$. The approach taken in $[8,9]$ is to enumerate all combinations $L$ with the Properties 1-2, which will give the positions of local minima $x^{*}$ and their values $d$ by using Properties 3-4.

From (23), combinations of $L$-matrices can be built incrementally, by taking initially the first $n+1$ support vectors (which yields the unique combination $L=\left\{l^{1}, l^{2}, \ldots, l^{n+1}\right\}$ ), and then adding one new support vector at a time. Suppose, we have already identified the local minima of $f_{k}$, i.e., all the required combinations. When we add another support vector $l^{k+1}$, we can inherit most of the local minima of $f_{k+1}$ (a few will be lost since Property 2 may fail with $l^{k+1}$ playing the role of $l^{r}$ ), and we only need to add a few new local minima, that are new combinations necessarily involving $l^{k+1}$. These new combinations are simple modifications of those combinations because Property 2 fails with $l^{r}=l^{k+1}$.

When ECAM is applied for solving the global optimization subproblem (4) at step $r$ of ERA, the procedure finishes when $f_{\text {best }}-d^{*}>\beta$ so, a $\beta$-global optimal solution is obtained. 
Remark 13 Notice that the transformation of variables

$$
\begin{aligned}
& \text { 1) } \bar{x}_{i}=x_{i}-a_{i}, i=1, \ldots, n, d=\sum_{i=1}^{n}\left(b_{i}-a_{i}\right) \text { with } \bar{x}_{i} \geq 0 \text { and } \sum_{i=1}^{n} \bar{x}_{i} \leq d \\
& \text { 2) } z_{i}=\frac{\bar{x}_{i}}{d}, i=1, \ldots, n, z_{n+1}=\sum_{i=1}^{n} z_{i},
\end{aligned}
$$

allows us to replace the program

$$
\min \{f(x): x \in[a, b]\}
$$

by the following one:

$$
\min \left\{g\left(z_{1}, \ldots, z_{n+1}\right):\left(z_{1}, \ldots, z_{n+1}\right) \in X\right\},
$$

where $S$ denotes the unit simplex in $\mathbb{R}^{n+1}$.

\section{Appendix: Performance profiles}

In this paper we compare, on the one hand, 8 implementations of the classical fixed step relaxation algorithm corresponding to 8 choices of $\lambda$ on a battery of 27 feasibility problems and, on the other hand, 5 implementations of the new relaxation algorithm with variable step size corresponding to 5 choices of $v$ on the same set of test problems. Denote by $\mathcal{S}$ the set of implementations to be compared, so that the cardinality of $\mathcal{S}$, denoted by size $\mathcal{S}$ is 8 and 5 for the classic and for the new relaxation algorithms, respectively. Denote also by $\mathcal{P}$ the set of test feasibility problems, with size $\mathcal{P}=27$ for both algorithms.

The notion of performance profile [20] allows us to compare the performance of the implementations from $\mathcal{S}$ on $\mathcal{P}$. For each pair $(p, s) \in \mathcal{P} \times \mathcal{S}$ we define

$f_{p, s}:=$ number of function evaluations required to solve problem $p$ by solver $s$.

Consider a fixed problem $p \in \mathcal{P}$. The performance of a solver $s \in \mathcal{S}$ able to solve $p$ is compared with the best performance of any solver of $\mathcal{S}$ on the same problem through the performance ratio

$$
r_{p, s}:=\frac{f_{p, s}}{\min \left\{f_{p, s}: s \in \mathcal{S}\right\}} \geq 1 .
$$


Obviously, $r_{p, s}=1$ means that $s$ is a winner for $p$, as it is at least as good, for solving $p$, as any other solver of $\mathcal{S}$. For any solver $s$ unable to solve problem $p$ we define $r_{p, s}=r_{M}$, where $r_{M}$ denotes an arbitrary scalar such that

$$
r_{M}>\max \left\{r_{p, s}: s \text { solves } p,(p, s) \in \mathcal{P} \times \mathcal{S}\right\} .
$$

The evaluation of the overall performance of $s \in \mathcal{S}$ is based on the stepwise non-decreasing function $\rho_{s}: \mathbb{R}_{+} \rightarrow[0,1]$, called performance profile of $s$, defined as follows:

$$
\rho_{s}(t)=\frac{\operatorname{size}\left\{p \in \mathcal{P}: r_{p, s} \leq t\right\}}{\operatorname{size} \mathcal{P}}, t \geq 0 .
$$

Obviously, $\rho_{s}(t)=0$ for all $t \in\left[0,1\left[\right.\right.$ and $\rho_{s}(1)$ is the relative frequency (which could be interpreted as a probability when $p$ is taken at random from $\mathcal{P})$ of wins of solver $s$ over the rest of the solvers. We say in brief that $\rho_{s}(1)$ is the probability of win for $s$.

Analogously, for $t>1, \rho_{s}(t)$ represents the probability for solver $s \in \mathcal{S}$ that a performance ratio $r_{p, s}$ is within a factor $t \in \mathbb{R}$ of the best possible ratio, so that $\rho_{s}$ can be interpreted as a distribution function and the number

$$
\rho_{s}^{*}:=\lim _{t \searrow_{M}} \rho_{s}(t)
$$

as the probability of solving a problem of $\mathcal{P}$ with $s \in \mathcal{S}$.

\section{Acknowledgments}

The authors are grateful to the referees for their constructive comments and helpful suggestions which have contributed to the final preparation of the paper.

\section{References}

[1] Agmon, S. (1954). The relaxation method for linear inequalities. Can. J. Math. 6, 382-392.

[2] Auslender, A., Ferrer, A., Goberna, M.A. \& López, M.A. (2015). Comparative study of RPSALG algorithm for convex semi-infinite programming. Comput. Optim. Appl. 60, 59-87. 
[3] Bagirov A.M. \& Rubinov A.M. (2000). Global minimization of increasing positively homogeneous functions over the unit simplex. Ann. Oper. Res. 98, 171-187.

[4] Bagirov, A.M. \& Rubinov, A.M. (2001). Modified versions of the cutting angle method. In: N. Hadjisavvas \& P.M. Pardalos (Eds.), Advances in convex analysis and global optimization (pp. 245-268). Dordrecht: Kluwer.

[5] Bartle, R.G. (1964). The elements of real analysis. New York: Wiley.

[6] Basu, A., De Loer, J.A. \& Junod, M. (2013). On Chubanov's method for Linear Programming. INFORMS J. Comput. 26, 336-350.

[7] Beliakov, G. (2003). Geometry and combinatorics of the cutting angle method. Optimization 52, 379-394.

[8] Beliakov, G. (2004). Cutting angle method. A tool for constrained global optimization. Optim. Method. Softw. 19, 137-151.

[9] Beliakov, G. (2005). A review of applications of the cutting angle method. In: A.M. Rubinov \& V. Jeyakumar (Eds.), Continuous optimization (pp. 209-248). New York: Springer.

[10] Beliakov, G. (2008). Extended cutting angle method of global optimization. Pacific J. Optim. 4, 153-176

[11] Beliakov, G. \& Ferrer, A. (2010). Bounded lower subdifferentiability optimization techniques: applications. J. Global Optim. 47, 211-231.

[12] Ben-Tal, A., El Ghaoui, L. \& Nemirovski, A. (2009). Robust optimization. Princeton: Princeton U.P.

[13] Ben-Tal, A. \& Nemirovski, A. (1999). Robust solutions of uncertain linear programs. Oper. Res. Lett. 25 (1999), no. 1, 1-13.

[14] Betke, U. (2004), Relaxation, new combinatorial and polynomial algorithms for the Linear Feasibility Problem. Discrete Comput. Geom. 32, 317-338.

[15] Borwein, J.M. \& Tam, M.K. (2014). A Cyclic Douglas-Rachford Iteration Scheme. J Optim Theory Appl. 160, 1-29. 
[16] Cánovas, M.J., López, M.A., Parra, J. \& Toledo, F.J. (2005). Distance to ill-posedness and the consistency value of linear semi-infinite inequality systems. Math. Programming 103A, 95-126.

[17] Cheney, E.W. \& Goldstein, A.A. (1959). Newton method for convex programming and Tchebycheff approximation. Numer. Math. 1, 253268.

[18] Combettes, P.L. (1996). The convex feasibility problem in image recovery. In In: Hawkes P (ed.), Advances in Imaging and Electron Physics, vol. 95, pp. 155-270, New York: Academic Press.

[19] Dinh, N., Goberna, M.A. \& López, M.A. (2006). From linear to convex systems: Consistency, Farkas Lemma and applications. J. Convex Anal. $13,279-290$.

[20] Dolan, E.D. \& Moré, J.J. (2002). Benchmarking optimization software with performance profiles. Math. Programming 91, 201-213.

[21] Eriksson, K., Estep, D. \& Johnson, C. (2004). Applied mathematics: body and soul. Berlin: Springer.

[22] Ferrer, A. \& Miranda, E. (2013). Random test examples with known minimum for convex semi-infinite programming problems. E-prints UPC. http://hdl.handle.net/2117/19118. Accessed 28 February 2015.

[23] Goberna, M.A., Jeyakumar, V., Li, G. \& Vicente-Pérez, J. (2014). Robust solutions of uncertain multi-objective linear semi-infinite programming. SIAM J. Optim. 24, 1402-1419.

[24] Goberna, M.A., Jeyakumar, V., Li, G. \& Vicente-Pérez, J. (2015). Robust solutions to multi-objective linear programs with uncertain data, European J. Oper. Res. 242, 730-743.

[25] Goberna, M.A. \& López, M.A. (1998). Linear semi-infinite optimization. Chichester: Wiley.

[26] Goberna, M.A. \& López, M.A. (2014). Post-optimal analysis in linear semi-infinite optimization, Springer Briefs, New York: Springer. 
[27] González-Gutiérrez, E., Rebollar, L.A. \& Todorov M.I. (2011). Rate of convergence of a class of numerical methods solving linear inequality systems. Optimization 60, 947-957.

[28] González-Gutiérrez, E., Rebollar, L.A. \& Todorov M.I. (2011). Under and over projection methods for solving linear inequality systems. Comptes Rendus de la Academie Bulgare des Sciences 64, 785-790.

[29] González-Gutiérrez, E., Rebollar, L.A. \& Todorov M.I. (2012). Relaxation methods for solving linear inequality systems: converging results. Top 20, 426-436.

[30] González-Gutiérrez, E. \& Todorov, M.I. (2012). A relaxation method for solving systems with infinitely many linear inequalities. Optimization Letters 6, 291-298.

[31] González-Gutiérrez E. \& Todorov, M.I. (2014). Generalized step iteration in the relaxation method for the feasibility problem. IMPA, Rio de Janeiro, BR, Preprint serie D113/2014.

https://institucional.impa.br/preprint/lista.action?serie $=4$

[32] Horst, R., Pardalos, P. \& Thoai, N.V. (2000). Introduction to global optimization (First ed.). Dordrecht: Kluwer.

[33] Hu, H. (1994). A Projection method for solving infinite systems of linear inequalities. In: D-Z. Du \& J. Sun (Eds.), Advances in optimization and approximation (pp 186-194). Dordrecht: Kluwer.

[34] Jeroslow, R.G. (1979). Some relaxation methods for linear inequalities, Cahiers du Cero 21, 43-53.

[35] Kelley, J.E., Jr. (1960). The cutting-plane method for solving convex programs. J. Soc. Indust. Appl. Math. 8, 703-712.

[36] Rubinov, A.M. (2000). Abstract convexity and global optimization. Dordrecht/Boston: Kluwer. 